\title{
Tetrahydrofuran Ca-Tetrasubstituted Amino Acids: Two consecutive $\beta$-Turns in Crystalline linear Tripeptide
}

\author{
Prantik Maity, Manfred Zabel and Burkhard König* \\ Institut für Organische Chemie, Universität Regensburg, D-93040 Regensburg, Germany
}

\section{Supporting Information}

\section{Table of contents}

1. Experimental procedures and compound characterisation

2. X-ray structure analyses

3. Copies of ${ }^{1} \mathrm{H}$ NMR spectra

4. Temperature dependent NMR \& ROESY spectra
Pages

S 1

S 11

S 13

S 35

\section{Experimental procedures and compound characterisation}

General: Melting points were determined on a melting point apparatus and are uncorrected. Specific rotations were measured on a polarimeter using a $10 \mathrm{~cm}$ cell. NMR spectra were recorded in $\mathrm{CDCl}_{3}$ at $300 \mathrm{MHz}\left({ }^{1} \mathrm{H}\right)$ or $75 \mathrm{MHz}\left({ }^{13} \mathrm{C}\right)$ unless stated otherwise. Structural assignments are based on DEPT and COSY experiments where applicable. The multiplicity of the carbon atoms is given as $(+)=\mathrm{CH}_{3}$ or $\mathrm{CH},(-)=\mathrm{CH}_{2}$ and $\left(\mathrm{C}_{\text {quat }}\right)$ for quaternary carbon atoms. Analytical TLC plates (silica gel $\left.60 \mathrm{~F}_{254}\right)$ and silica gel $60(70-230$ or 230-400 mesh) for column chromatography (CC) were purchased from Merck. Visualization of spots by UV light and/or staining with phosphomolybdate or ninhydrin, both in ethanol. $\mathrm{DMF}, \mathrm{CH}_{3} \mathrm{CN}$, THF, and $\mathrm{Et}_{2} \mathrm{O}$ were dried by standard procedures and stored over molecular sieves or Na. PE means petrol ether with a boiling range of 70-90 ${ }^{\circ} \mathrm{C}$. All other solvents and chemicals were of reagent grade and used with out further purification. 
tert-Butyl methionine : Racemic methionine (10 g, $67 \mathrm{mmol}), 1,4$-dioxane $(40 \mathrm{~mL})$ and 1.25 $\mathrm{M}$ aqueous $\mathrm{NaOH}(53 \mathrm{~mL})$ were stirred and cooled to $6^{\circ} \mathrm{C}$. Then a solution of di-tertbutyl-dicarbonate $(15.4 \mathrm{~g}, 70.4 \mathrm{mmol})$ in 1,4-dioxane $(12 \mathrm{~mL})$ was added over $15 \mathrm{~min}$. The cooling bath was removed and the reaction stirred for $3.5 \mathrm{~h}$. The dioxane was removed in vacuo, the remaining mixture was diluted with $1 \mathrm{M}$ aqueous $\mathrm{KHSO}_{4}(68 \mathrm{~mL})$ and extracted with EtOAc $(1 \times 40,1 \times 25 \mathrm{~mL})$. The combined organic layers were washed with water $(24$ $\mathrm{mL})$, brine $(4 \mathrm{~mL})$ and dried over $\mathrm{MgSO}_{4}$. The solvent was removed to give pure tert-butyl methionine as colourless liquid (15 g, 90\%).

\section{tert-Butyl)-4-(2-(tert-butoxycarbonylamino methylthio)butanoate (rac-2a)}<smiles>CCCCC(NC(CCSC)C(=O)OC)C(=O)OC</smiles>

To a cooled $\left(0^{\circ} \mathrm{C}\right)$ solution of tert-butyl methionine $(2 \mathrm{~g}, 8 \mathrm{mmol})$, DMAP $(0.08 \mathrm{~g}, 0.67$ mmol $)$ and tert-butanol $(0.71 \mathrm{~g}, 9.6 \mathrm{mmol})$ in dry $\mathrm{CH}_{2} \mathrm{Cl}_{2}(20 \mathrm{~mL}) \mathrm{N}, \mathrm{N}^{\prime}-$ dicyclohexylcarbodiimide $(2.15 \mathrm{~g}, 10.4 \mathrm{mmol})$ was added with stirring and the reaction mixture was stirred at $0^{\circ} \mathrm{C}$ for $2 \mathrm{~h}$. After stirring for $12 \mathrm{~h}$ at room temperature, the precipitated dicyclohexylurea was filtered off and washed with DCM $(2 \times 10 \mathrm{~mL})$. The organic layer was washed with $1 \mathrm{M} \mathrm{HCl}(2 \times 5 \mathrm{~mL})$, a saturated aqueous solution of $\mathrm{NaHCO}_{3}(2 \times 10 \mathrm{~mL})$ and water $(2 \times 5 \mathrm{~mL})$ and dried over $\mathrm{MgSO}_{4}$. Then the solvent was evaporated in vacuo, and the crude product was purified by column chromatography $\left(\mathrm{SiO}_{2}, \mathrm{PE}:\right.$ diethyl ether $\left.4: 1\right)$ to give $2 \mathrm{~g}$ of compound 2 ( $82 \%$ yield). $R_{\mathrm{f}}=0.20$ (diethyl ether : $\mathrm{PE}=1: 4$ )

${ }^{1} \mathrm{H}$ NMR $\left(300 \mathrm{MHz}, \mathrm{CDCl}_{3}\right) \delta=1.45(\mathrm{~s}, 18 \mathrm{H}), 1.99$ (m, 1H, -CHH-), $2.11(\mathrm{~s}, 3 \mathrm{H}), 2.15$ (m, $1 \mathrm{H},-\mathrm{CH} H-), 2.58(\mathrm{~m}, 2 \mathrm{H}), 4.45(\mathrm{bs}, 1 \mathrm{H}), 5.25(\mathrm{bs}, 1 \mathrm{H}) .-{ }^{13} \mathrm{C} \mathrm{NMR}\left(75.5 \mathrm{MHz}, \mathrm{CDCl}_{3}\right), \delta=$ $27.64(+, 3 \mathrm{C}), 27.99(+, 3 \mathrm{C}), 28.30(-), 30.40(-), 53.38(+), 65.83(+), 72.08\left(\mathrm{C}_{\text {quat }}\right), 77.28$ $\left(\mathrm{C}_{\text {quat }}\right), 155.33\left(\mathrm{C}_{\text {quat }}\right), 171.36\left(\mathrm{C}_{\text {quat }}\right)$. MS [ESI H $\left.{ }_{2} \mathrm{O} / \mathrm{AcN}\right]: \mathrm{m} / \mathrm{z}(\%)=305.5\left[\mathrm{MH}^{+}\right](100)$.

\section{(4-tert-Butoxy 3-(tert-butoxycarbonylamino)-4-oxobutyl)dimethylsulfonium iodide} (rac-3a)

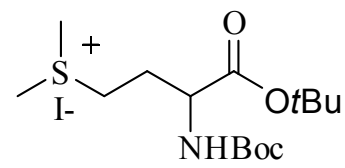


Compound rac-2 (10 g, $32.7 \mathrm{mmol})$, methyl iodide (46.86 g, $0.33 \mathrm{~mol})$ and acetone (10 $\mathrm{mL}$ ) were stirred at room temperature for $3 \mathrm{~d}$ in the dark. During this time a white precipitate was formed. After cooling in an ice bath for $4 \mathrm{~h}$, the precipitate was filtered off and washed with chilled solvent $\left(0^{\circ} \mathrm{C}\right.$ temp. diethyl ether : acetone $\left.9: 1,2 \times 10 \mathrm{~mL}\right)$. This solid was dried in vacuo yielding compound rac-3 (11.4 g, 78\%) in analytical pure form.

${ }^{1} \mathrm{H}$ NMR (300 MHz, $\left.\mathrm{CDCl}_{3}\right) \delta=1.45$ (s, 9H), 1.47 (s, 9H), 2.29 (m, 1H,-CHH-), 2.32 (m, 1H, -CHH), 3.30 (d, 6H), 3.70 (m, 1H, -SCHH-), 3.75 (m, 1H, -SCHH-), 4.15 (bs, 1H), 5.70 (bs, 1H). $-{ }^{13} \mathrm{C}$ NMR $\left(75.5 \mathrm{MHz}, \mathrm{CDCl}_{3}\right) \delta=15.48(+), 28.00(+), 28.05(+), 29.92(-), 32.56$ $(-), 53.39(+), 80.63\left(\mathrm{C}_{\text {quat }}\right), 82.12\left(\mathrm{C}_{\text {quat }}\right), 169.65\left(\mathrm{C}_{\text {quat }}\right), 171.00\left(\mathrm{C}_{\text {quat }}\right) . \mathrm{MS}$ [ESI, $\left.\mathrm{H}_{2} \mathrm{O} / \mathrm{AcN}\right]$ : $\mathrm{m} / \mathrm{z}(\%)=320.1\left[\mathrm{M}^{+}-\mathrm{I}\right](100)$

tert-Butyl 3-(tert-butoxycarbonylamino)-2-(4-nitrophenyl)tetrahydrofuran-3-carboxylate $(r a c-4 b)$

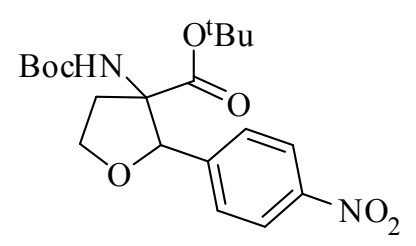

Yield $=35 \%$, using $\mathrm{KOH}$ as base; $R_{\mathrm{f}}=0.18$ (diethyl ether : $\mathrm{PE}=3: 17$ ), m.p. $=146-148^{\circ} \mathrm{C}$.

${ }^{1} \mathrm{H}$ NMR $\left(300 \mathrm{MHz}, \mathrm{CDCl}_{3}\right) \delta=1.30(\mathrm{~s}, 9 \mathrm{H}), 1.50(\mathrm{~s}, 9 \mathrm{H}), 2.55(\mathrm{~m}, 1 \mathrm{H},-\mathrm{CHH}-), 2.80(\mathrm{~m}$, 1H, - $\mathrm{CH} H-), 4.05$ (m, 1H, -OCHH-), 4.25 (m, 1H, -OCHH-), 4.45 (bs, 1H), 5.25 (bs, 1H), $7.55(\mathrm{~d}, J=9.26 \mathrm{~Hz}, 2 \mathrm{H}), 8.23(\mathrm{~d}, J=9.26 \mathrm{~Hz}, 2 \mathrm{H}) .-{ }^{13} \mathrm{C} \mathrm{NMR}\left(75.5 \mathrm{MHz}, \mathrm{CDCl}_{3}\right) \delta=$ $27.92(+), 28.12(+), 35.80(-), 67.04(-), 68.09(+), 82.65\left(\mathrm{C}_{\text {quat }}\right), 84.87\left(\mathrm{C}_{\text {quat }}\right), 123.44(+)$, $128.08\left(\mathrm{C}_{\text {quat }}\right), 147.88(+), 154.45\left(\mathrm{C}_{\text {quat }}\right), 170.47\left(\mathrm{C}_{\text {quat }}\right), 171.5\left(\mathrm{C}_{\text {quat }}\right) . \quad-\mathrm{MS}$ [ESI;

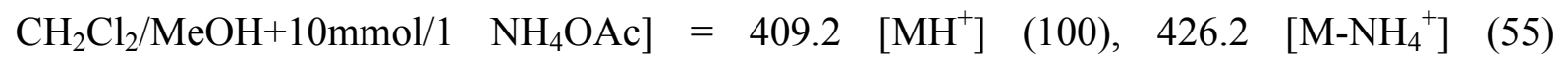
- IR (KBr): $\widetilde{v} \mathrm{~cm}^{-1}=3375,2981,2934,2877,1730,1603,1504,1452$. Anal. calcd. For $\mathrm{C}_{20} \mathrm{H}_{28} \mathrm{~N}_{2} \mathrm{O}_{7}$ (408.45): C 58.81, H 6.91, N 6.86, found C 58.45, H 7.07, N 6.85.

tert-Butyl 2-(3-bromophenyl)-3-(tert-butoxycarbonylamino)tetrahydrofuran-3-carboxylate (rac-4f)<smiles>CCCCNC1(C(=O)OCC(C)C)CCOC1c1cccc(Br)c1</smiles> 
Yield $=50 \%$, using $\mathrm{KOH}$ as a base; $R_{\mathrm{f}}=0.14$ (diethyl ether: $\mathrm{PE}=3: 17$ ).

${ }^{1} \mathrm{H}$ NMR $\left(300 \mathrm{MHz}, \mathrm{CDCl}_{3}\right) \delta=1.15$ (s, 9H), 1.48 (s, 9H), 2.59-2.71 (m, 2H), 4.18-4.36 $(\mathrm{m}, 2 \mathrm{H}), 5.07(\mathrm{bs}, 1 \mathrm{H}), 5.65(\mathrm{bs}, 1 \mathrm{H}), 7.13-7.23(\mathrm{~m}, 2 \mathrm{H}), 7.36-7.41(\mathrm{~m}, 1 \mathrm{H}), 7.53-7.55(\mathrm{~m}$, 1H). ${ }^{13} \mathrm{C}$ NMR $\left(75.5 \mathrm{MHz}, \mathrm{CDCl}_{3}\right) \delta=27.43(+), 28.41(+), 35.76(-), 67.97(-), 69.59(+)$, $80.14\left(\mathrm{C}_{\text {quat }}\right), 82.70\left(\mathrm{C}_{\text {quat }}\right), 84.02\left(\mathrm{C}_{\text {quat }}\right), 122.27(+), 124.67(+), 129.35\left(\mathrm{C}_{\text {quat }}\right), 129.54(+)$, $130.88\left(\mathrm{C}_{\text {quat }}\right), 140.05(+), 154.34\left(\mathrm{C}_{\text {quat }}\right), 170.03\left(\mathrm{C}_{\text {quat }}\right)$. - $\mathrm{MS}\left[\mathrm{ESI} ; \mathrm{CH}_{2} \mathrm{Cl}_{2} / \mathrm{MeOH}+10\right.$ mmol/1 NH $\mathrm{NHAc}_{4} \mathrm{OA} 442.2\left[\mathrm{MH}^{+}\right]$(30), 459.0. $\left[\mathrm{MNH}_{4}{ }^{+}\right]$(30), $403.0\left[\mathrm{M}-\mathrm{NH}_{4}{ }^{+}-\mathrm{C}_{4} \mathrm{H}_{8}\right]$ (100) - IR (KBr): $\widetilde{v} \mathrm{~cm}^{-1}=3361,3070$. 2978, 2621, 2534, 2199, 1698, 1570, 1479, 1393.

tert-Butyl 3-(tert-butoxycarbonylamino)-2-(4-fluro-phenyl)tetrahydrofuran-3-carboxylate $(\mathrm{rac}-\mathbf{4 g})$

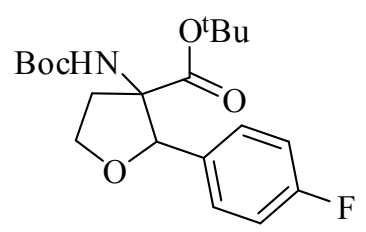

Yield $=63 \%$, using $\mathrm{KOH}$ as a base. $R_{\mathrm{f}}=0.12$ (diethyl ether : $\left.\mathrm{PE}=1: 4\right)$, m.p $=89-92^{\circ} \mathrm{C}$.

${ }^{1} \mathrm{H}$ NMR $\left(300 \mathrm{MHz}, \mathrm{CDCl}_{3}\right) \delta=1.09(\mathrm{~s}, 9 \mathrm{H}), 1.49$ (s, 9H), 2.67-2.78 (m, 2H), 4.16-4.33(m, 2H), $5.00(\mathrm{bs}, 1 \mathrm{H}), 5.71(\mathrm{bs}, 1 \mathrm{H}), 7.00(\mathrm{~m}, 2 \mathrm{H}), 7.42(\mathrm{~m}, 2 \mathrm{H}) .-{ }^{13} \mathrm{C} \mathrm{NMR}\left(75.5 \mathrm{MHz}, \mathrm{CDCl}_{3}\right)$ $\delta=27.44(+), 28.40(+), 35.80(-), 67.91(-), 69.63(+), 80.13\left(\mathrm{C}_{\text {quat }}\right), 82.62\left(\mathrm{C}_{\text {quat }}\right), 84.42$ $\left(\mathrm{C}_{\text {quat }}\right), 114.99(+), 127.98(+), 131.01\left(\mathrm{C}_{\text {quat }}\right), 154.38\left(\mathrm{C}_{\text {quat }}\right), 160\left(\mathrm{C}_{\text {quat }}\right), 170.03\left(\mathrm{C}_{\text {quat }}\right) .-\mathrm{MS}$ [ESI; $\left.\mathrm{CH}_{2} \mathrm{Cl}_{2} / \mathrm{MeOH}+10 \mathrm{mmol} \mathrm{NH}{ }_{4} \mathrm{OAc}\right] .=.382 .1\left[\mathrm{MH}^{+}\right](40) ; 343.1\left[\mathrm{MNH}_{4}{ }^{+}-\mathrm{C}_{4} \mathrm{H}_{8}\right],(100)$; 326.1[ $\left.\mathrm{MH}^{+}-\mathrm{C}_{4} \mathrm{H}_{8}\right],(60)$. - IR (KBr): $\widetilde{v} \mathrm{~cm}^{-1}=3377,2980,2863,2199,1710,1606,1495$, 1447.

tert-Butyl 3-(tert-butoxycarbonylamino)-2-(4-cyano-phenyl)tetrahydrofuran-3-carboxylate $(\mathrm{rac}-4 \mathrm{~h})$

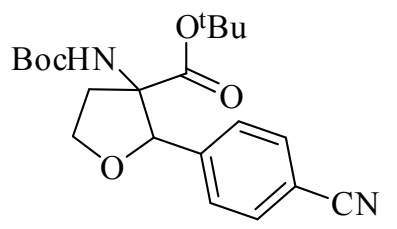


cis/trans $=9: 1$. Yield $=55 \%$, using $\mathrm{KOH}$ as a base; $R_{\mathrm{f}}=0.14$ (diethyl ether $:$ PE. $\left.=3: 17\right)$, m.p. $=128-130^{\circ} \mathrm{C}$.

Resonance signals of the trans isomer: ${ }^{1} \mathrm{H}$ NMR $\left(300 \mathrm{MHz}, \mathrm{CDCl}_{3}\right) \delta=1.06(\mathrm{~s}, 9 \mathrm{H}), 1.46(\mathrm{~s}$, 9H), 2.71-2.49 (m, 2H), 4.31-4.20 (m, 2H), 5.02 (bs, 1H), 5.65 (bs, 1H), 7.45 (d, J = 8.78 Hz, $2 \mathrm{H}), 7.58(\mathrm{~d}, J=8.78 \mathrm{~Hz}, 2 \mathrm{H})$. Resonance signals of the cis isomer: ${ }^{1} \mathrm{H}$ NMR $(300 \mathrm{MHz}$, $\left.\mathrm{CDCl}_{3}\right) \delta=1.28(\mathrm{~s}, 9 \mathrm{H}), 1.47(\mathrm{~s}, 9 \mathrm{H}), 2.56(\mathrm{~m}, 1 \mathrm{H},-\mathrm{CHH}-), 2.77(\mathrm{~m}, 1 \mathrm{H},-\mathrm{CH} H-), 4.03(\mathrm{~m}$, 1H,-OCHH-), 4.23 (m, 1H, -OCH H-), 4.38 (bs, 1H), 5.15 (bs, 1H), 7.45 (d, J=8.78 Hz, 2H), $7.64(\mathrm{~d}, J=8.78 \mathrm{~Hz}, 2 \mathrm{H}) \cdot-{ }^{13} \mathrm{C}$ NMR $\left(75.5 \mathrm{MHz}, \mathrm{CDCl}_{3}\right) \delta=27.40(+), 28.40(+), 36.08(-)$, $68.07(-), 69.51,80.31\left(\mathrm{C}_{\text {quat }}\right), 82.95\left(\mathrm{C}_{\text {quat }}\right), 83.52(+), 111.47\left(\mathrm{C}_{\text {quat }}\right), 118.78\left(\mathrm{C}_{\text {quat }}\right), 126.13$ $(+), \quad 131.71(+), \quad 143.47 \quad\left(\mathrm{C}_{\text {quat }}\right), \quad 154.20 \quad\left(\mathrm{C}_{\text {quat }}\right), \quad 170.00 \quad\left(\mathrm{C}_{\text {quat }}\right) . \quad-\quad$ MS $\quad$ [ESI; $\left.\mathrm{CH}_{2} \mathrm{Cl}_{2} / \mathrm{MeOH}+10 \mathrm{mmol} / 1 \quad \mathrm{NH}_{4} \mathrm{OAc}\right]=389.3\left[\mathrm{MH}^{+}\right](30), 406.3\left[\mathrm{M}-\mathrm{NH}_{4}^{+}\right]$(45), $350.3[\mathrm{M}-$ $\left.\mathrm{NH}_{4}{ }^{+}-\mathrm{C}_{4} \mathrm{H}_{8}\right]$ (56). - IR (KBr): $\widetilde{v} \mathrm{~cm}^{-1}=3357,2982,2910,2877,1698,1613,1524,1425$. Anal. calcd. For $\mathrm{C}_{21} \mathrm{H}_{28} \mathrm{~N}_{2} \mathrm{O}_{5}$ (388.14): .C 64.93, H 7.27, N 7.21, found C 64.87, H 7.54, N 7.07 .

tert-Butyl 3-(tert-butoxyxycarbonylamino)-2-m-tolyltetrahydro-furan-3-carboxylate (rac-4i)

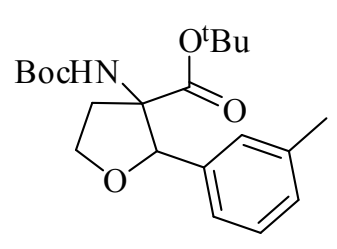

Yield $=55 \%$, using $\mathrm{KOH}$ as a base; $R_{\mathrm{f}}=0.14$ (diethyl ether : $\mathrm{PE}=3: 17$ ).

${ }^{1} \mathrm{H}$ NMR $\left(300 \mathrm{MHz}, \mathrm{CDCl}_{3}\right) \delta=1.00(\mathrm{~s}, 9 \mathrm{H}), 1.40(\mathrm{~s}, 9 \mathrm{H}), 2.22(\mathrm{~s}, 3 \mathrm{H}), 2.64-2.78(\mathrm{~m}, 1 \mathrm{H},-$ $\mathrm{CHH}-), 2.72(\mathrm{~m}, 1 \mathrm{H},-\mathrm{CH} H-), 4.13(\mathrm{~m}, 1 \mathrm{H},-\mathrm{OCHH}-), 4.24(\mathrm{~m}, 1 \mathrm{H},-\mathrm{OCH} H) 4.89$ (bs, 1H, $\mathrm{CH}-), 5.60$ (bs, 1H, NH-), 6.90-7,14 (m, 5H). ${ }^{13} \mathrm{C} \mathrm{NMR}\left(75.5 \mathrm{MHz}, \mathrm{CDCl}_{3}\right) \delta=21.12(+)$, $27.41(+), 29.94(+), 35.73(-), 67.78(-), 69.74(+), 82.05\left(\mathrm{C}_{\text {quat }}\right), 82.07\left(\mathrm{C}_{\text {quat }}\right), 85.74\left(\mathrm{C}_{\text {quat }}\right)$, $126.21(+), 128.61(+), 134.38\left(\mathrm{C}_{\text {quat }}\right), 137.63\left(\mathrm{C}_{\text {quat }}\right), 154.57\left(\mathrm{C}_{\text {quat }}\right), 170.07\left(\mathrm{C}_{\text {quat }}\right) .-\mathrm{MS}$

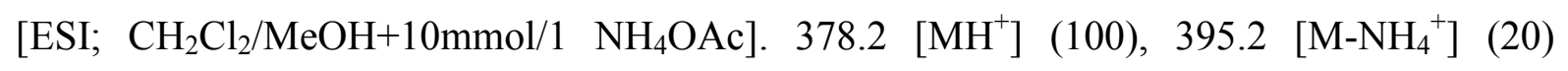
- IR (KBr): $\widetilde{v} \mathrm{~cm}^{-1}=3357,2978,2930,2870,2783,2199,1703,1610,1514,1450$. 

ylate $(r a c-4 j)$

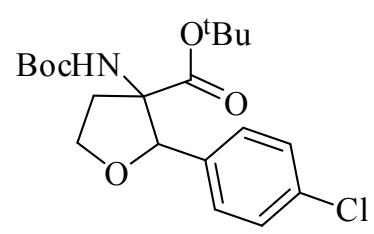

Yield $=55 \%$, using $\mathrm{KOH}$ as a base; $R_{\mathrm{f}}=0.13$ (diethyl ether $: \mathrm{PE}=3: 17$ ) m.p $=107-110^{\circ} \mathrm{C}$.

${ }^{1} \mathrm{H}$ NMR (300 MHz, $\left.\mathrm{CDCl}_{3}\right) \delta=1.12$ (s, 9H), 1.47 (s, 9H), 2.60-2.65 (m, 2H), 4.15-4.38 (m, 2H), $5.00(\mathrm{bs}, 1 \mathrm{H}), 5.60(\mathrm{bs}, 1 \mathrm{H}), 7.27(\mathrm{~m}, 4 \mathrm{H}) .-{ }^{13} \mathrm{C} \mathrm{NMR}\left(75.5 \mathrm{MHz}, \mathrm{CDCl}_{3}\right) \delta=27.41$ $(+), 29.94(+), 35.62(-), 67.90(-), 69.63\left(\mathrm{C}_{\text {quat }}\right), 82.52\left(\mathrm{C}_{\text {quat }}\right), 127.58(+), 128.08(+), 133.65$ $\left(\mathrm{C}_{\text {quat }}\right)$, $137.63\left(\mathrm{C}_{\text {quat }}\right), 154.57\left(\mathrm{C}_{\text {quat }}\right), 170.07$ ( $\left.\mathrm{C}_{\text {quat }}\right)$. - MS [ESI; $\mathrm{CH}_{2} \mathrm{Cl}_{2} / \mathrm{MeOH}+10 \mathrm{mmol} / 1$ $\left.\mathrm{NH}_{4} \mathrm{OAc}\right]=378.2\left[\mathrm{MH}^{+}\right](100), 395.2\left[{\mathrm{M}-\mathrm{NH}_{4}}^{+}\right](20)-\mathrm{IR}(\mathrm{KBr}): \widetilde{v} \mathrm{~cm}^{-1}=3375,2981$, 2934, 2877, 1730, 1603, 1504, 1452. Anal. calcd. For $\mathrm{C}_{20} \mathrm{H}_{28} \mathrm{ClNO}_{5}$ (397): C 60.37, H 7.09, N 3.51, found C 60.45, H 7.07, N 3.53.

tert-Butyl 3-(tert-butoxycarbonylamino)-2-(naphthalene-2-ly)tetrahydrofuran-3-carboxylate $(r a c-4 k)$

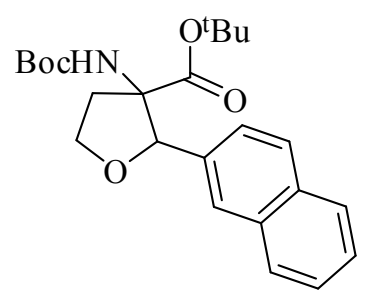

Yield $=55 \%$, using $\mathrm{KOH}$ as a base; $R_{\mathrm{f}}=0.22$ (diethyl ether $\left.: \mathrm{PE}=1: 4\right)$, m.p $=140-143^{\circ} \mathrm{C}$.

${ }^{1} \mathrm{H}$ NMR $\left(300 \mathrm{MHz} \mathrm{CDCl}_{3}\right) \delta=1.00(\mathrm{~s}, 9 \mathrm{H}), 1.45$ (s, 9H), 2.50-2.80 (m, 2H), $4.23(\mathrm{~m}, 1 \mathrm{H},-$ OCHH-), 4.33 (m, 1H, -OCHH-), 5.02 (bs, 1H), 5.60 (bs, 1H), 7.45 (m, 3H), 7.80(m, 4H). ${ }^{13} \mathrm{C}$ NMR (75.5 MHz, $\left.\mathrm{CDCl}_{3}\right) \delta=27.92(+), 28.12(+), 35.83(-), 67.04(-), 82.65\left(\mathrm{C}_{\text {quat }}\right)$, $84.87\left(\mathrm{C}_{\text {quat }}\right), 123.44(+), 128.08\left(\mathrm{C}_{\text {quat }}\right), 133.53(+), 137.52(+), 150.61(+), 154.54(+)$, $155.36\left(\mathrm{C}_{\text {quat }}\right), 170.09\left(\mathrm{C}_{\text {quat }}\right)$ - IR (KBr): $\widetilde{v} \mathrm{~cm}^{-1}=3360,2978,2931,2878,2199,1703$, 1504, 1454. - HRMS cald. for $\mathrm{C}_{24} \mathrm{H}_{31} \mathrm{NO}_{5}$ (413.2206) found $413.2205 \pm$.2. Anal. calcd. For $\mathrm{C}_{24} \mathrm{H}_{31} \mathrm{NO}_{5}$ (413): C 69.71, H 7.56, N 3.39, found C 69.51, H 7.74, N 3.30. 

(rac-4l)

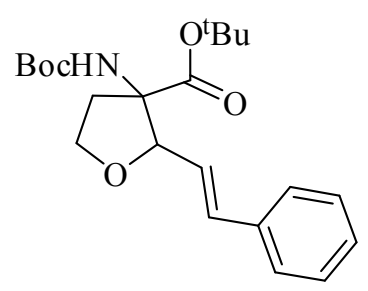

Yield $=53 \%$, using $\mathrm{KOH}$ as a base; $R_{\mathrm{f}}=0.14$ (diethyl ether $\left.: \mathrm{PE}=3: 17\right)$, m.p $=134-136^{\circ} \mathrm{C}$.

${ }^{1} \mathrm{H}$ NMR $\left(300 \mathrm{MHz}, \mathrm{CDCl}_{3}\right) \delta=1.39(\mathrm{~s}, 9 \mathrm{H}), 1.46(\mathrm{~s}, 9 \mathrm{H}), 2.46(\mathrm{~m}, 1 \mathrm{H},-\mathrm{CH} H-), 2.73-2.82$ (m, 1H, -CHH-), 4.07 (m, 1H, -OCHH-), 4.21 (m, 1H, -OCHH-), 4.44 (d, J=6.31 Hz, 1H), $5.30(\mathrm{bs}, 1 \mathrm{H}), 6.02-6.12(\mathrm{dd}, J=15.92 \mathrm{~Hz}, 7.14 \mathrm{~Hz}, 1 \mathrm{H}), 6.60-6.65(\mathrm{~d}, J=15.92 \mathrm{~Hz}, 1 \mathrm{H})$, 7.22-7.35 (m, 5H). - ${ }^{13} \mathrm{C}$ NMR (75.5 MHz, $\left.\mathrm{CDCl}_{3}\right) \delta=27.93(+), 28.35(+), 35.17(-), 67.61$ $(-), 69.5\left(\mathrm{C}_{\text {quat }}\right), 80.02\left(\mathrm{C}_{\text {quat }}\right), 82.17\left(\mathrm{C}_{\text {quat }}\right), 85.50(+), 124.27(+), 126.66(+), 128.00(+)$, $133.36(+), 136.13(+), 154.78\left(\mathrm{C}_{\text {quat }}\right), 169.92\left(\mathrm{C}_{\text {quat }}\right)$. - MS [ESI; $\mathrm{CH}_{2} \mathrm{Cl}_{2} / \mathrm{MeOH}+10 \mathrm{mmol} / 1$ $\left.\mathrm{NH}_{4} \mathrm{OAc}\right] .=390.2\left[\mathrm{MH}^{+}\right](100), 278\left[\mathrm{M}-2 \mathrm{xC}_{4} \mathrm{H}_{8}\right](35), 407.2\left[{\mathrm{M}-\mathrm{NH}_{4}}^{+}\right](15)-\mathrm{IR}(\mathrm{KBr}): \widetilde{v}$ $\mathrm{cm}^{-1}=3375,2981,2934,2877,1730,1603,1504,1452$. Anal. calcd. For $\mathrm{C}_{22} \mathrm{H}_{31} \mathrm{NO}_{5}$ (389.49): C 67.84, H 8.02, N 3.60, found C 67.73, H 8.21, N 3.55.

tert-Butyl 3-(tert-butoxycabonylamino)octahydro-2, 2'-bifuran-3-carboxylate (rac-4m)

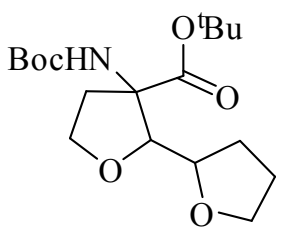

trans $/$ cis. $=3: 1$. Yield $=56 \%$, using $\mathrm{KO}^{\mathrm{t}} \mathrm{Bu}$ as a base; $R_{\mathrm{f}}=0.26$ (diethyl ether $: \mathrm{PE}=1: 4$ ), m.p. $=77-80^{\circ} \mathrm{C}$. Resonance signals of trans isomer: ${ }^{1} \mathrm{H} \mathrm{NMR}\left(300 \mathrm{MHz}, \mathrm{CDCl}_{3}\right) \delta=1.29(\mathrm{~s}$, 9H), $1.45(\mathrm{~s}, 9 \mathrm{H}), 2.50(\mathrm{~m}, 1 \mathrm{H},-\mathrm{CHH}), 2.87-2.99\left(\mathrm{~m}, 1 \mathrm{H},-\mathrm{CH} H_{-}\right), 4.13\left(\mathrm{~m}, 1 \mathrm{H},-\mathrm{OCH} H_{-}\right)$, 4.30 (m, 1H, -OCHH-), 4.89 (bs, 1H), 5.35 (bs, 1H), 6.32 (m, 2H), 7.36 (m, 1H). - ${ }^{1} \mathrm{H}$ NMR $\left(300 \mathrm{MHz}, \mathrm{C}_{6} \mathrm{D}_{6}\right) \delta=1.25(\mathrm{~s}, 9 \mathrm{H}), 1.43(\mathrm{~s}, 9 \mathrm{H}), 2.39-2.52(\mathrm{~m}, 1 \mathrm{H},-\mathrm{CHH}), 2.96-3.08(\mathrm{~m}, 1 \mathrm{H}$, - $\mathrm{CH} H-), 3.81-3.91$ (m, 1H, -OCHH-), 4.09-4.17 (m, 1H, -OCHH-), 4.76 (bs, 1H), 5.34 (bs, $1 \mathrm{H})$, 5.94-5.98 $(\mathrm{m}, 1 \mathrm{H}), 6.23-6.26(\mathrm{~m}, 1 \mathrm{H}), 6.92-6.95(\mathrm{~m}, 1 \mathrm{H})$. - Resonance signals of the $c$ is isomer: ${ }^{1} \mathrm{H}$ NMR $\left(300 \mathrm{MHz}, \mathrm{CDCl}_{3}\right) \delta=1.39(\mathrm{~s}, 9 \mathrm{H}), 1.49(\mathrm{~s}, 9 \mathrm{H}), 2.56(\mathrm{~m}, 1 \mathrm{H},-\mathrm{CHH})$, 
2.78-2.85 (m,1H,-CHH-), 4.13 (m, 1H, -OCHH-), 4.30 (m, 1H, -OCHH-), 4.87 (bs, 1H), 5.30 (bs, 1H), $5.97(\mathrm{~m}, 2 \mathrm{H}), 6,98(\mathrm{~m}, 1 \mathrm{H})-{ }^{13} \mathrm{C} \mathrm{NMR}\left(75.5 \mathrm{MHz}, \mathrm{CDCl}_{3}\right) \delta=27.58(+), 28.33(+)$, $35.17(-), 68.12(-), 69.05\left(\mathrm{C}_{\text {quat }}\right), 80.15\left(\mathrm{C}_{\text {quat }}\right), 81.05(+), 81.93\left(\mathrm{C}_{\text {quat }}\right), 108.19(+), 110.32$ $(+), \quad 142.38 \quad(+), \quad 150.63 \quad\left(\mathrm{C}_{\text {quat }}\right), \quad 154.93 \quad\left(\mathrm{C}_{\text {quat }}\right), \quad 169.19 \quad\left(\mathrm{C}_{\text {quat }}\right) . \quad-$ $\mathrm{MS}\left[\mathrm{ESI} ; \mathrm{CH}_{2} \mathrm{Cl}_{2} / \mathrm{MeOH}+10 \mathrm{mmol} / 1 \quad \mathrm{NH}_{4} \mathrm{OAc}\right]=354.1\left[\mathrm{MH}^{+}\right](100), 371.2\left[{\mathrm{M}-\mathrm{NH}_{4}}^{+}\right]$(30).

- IR (KBr): $\widetilde{v} \mathrm{~cm}^{-1}=3357,3119,2978,2933,2868,2199,1737,1703,1514,1448$. Anal. calcd. For $\mathrm{C}_{18} \mathrm{H}_{27} \mathrm{NO}_{6}$ (353.42): C 61.17, H 7.70, N 3.96, found C 60.94, H 7.69, N 3.90.

\section{Benzyl 2-(tert-butoxycarbonylamino)-4-(methylthio)butanoate (rac-2b)}<smiles>CSCCC(N)C(=O)OCc1ccccc1</smiles>

${ }^{1} \mathrm{H}$ NMR $\left(300 \mathrm{MHz}, \mathrm{CDCl}_{3}\right) \delta=1.45$ (s, 18H), 1.99 (m, 1H, CHH-), $2.11(\mathrm{~s}, 3 \mathrm{H}), 2.15(\mathrm{~m}, 1 \mathrm{H}$, $\mathrm{CH} H), 2.58(\mathrm{~m}, 2 \mathrm{H}), 4.45(\mathrm{bs}, 1 \mathrm{H}), 5.25(\mathrm{bs}, 1 \mathrm{H}) .{ }^{13} \mathrm{C} \mathrm{NMR}\left(75.5 \mathrm{MHz}, \mathrm{CDCl}_{3}\right), \delta=27.64$ $(+), 27.99(+), 28.30(-), 30.40(-), 53.38(+), 65.83(+), 72.08\left(\mathrm{C}_{\text {quat }}\right), 77.28\left(\mathrm{C}_{\text {quat }}\right), 155.33$ $\left(\mathrm{C}_{\text {quat }}\right), 171.36\left(\mathrm{C}_{\text {quat }}\right) . \mathrm{MS}\left[\mathrm{ESI} \mathrm{H} \mathrm{H}_{2} \mathrm{O} / \mathrm{AcN}\right]: \mathrm{m} / \mathrm{z}(\%)=305.5\left[\mathrm{MH}^{+}\right](100)$

(4-(Benzyloxy)-3-(tert-butoxycarbonylamino)-4-oxobutyl)dimethylsulfonium iodide (rac-3b)

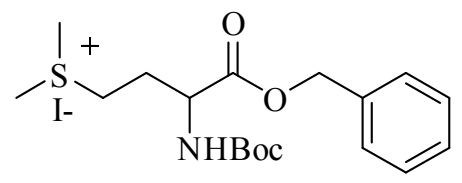

${ }^{1} \mathrm{H}$ NMR $\left(300 \mathrm{MHz}, \mathrm{CDCl}_{3}\right) \delta=1.45$ (s, 9H), 1.47 (s, 9H), $2.29(\mathrm{~m}, 1 \mathrm{H},-\mathrm{CHH}-), 2.32(\mathrm{~m}$, $1 \mathrm{H},-\mathrm{CH} H), 3.30(\mathrm{~d}, 6 \mathrm{H}), 3.70(\mathrm{~m}, 1 \mathrm{H},-\mathrm{SCHH}-), 3.75$ (m, -SCHH-), 4.15 (bs, 1H), 5.70 (bs, 1H). ${ }^{13} \mathrm{C} \mathrm{NMR}\left(75.5 \mathrm{MHz}, \mathrm{CDCl}_{3}\right) \delta=15.48(+), 28.00(+),, 28.05(+), 29.92(-), 32.56(-)$, $53.39(+), 80.63\left(\mathrm{C}_{\text {quat }}\right), 82.12\left(\mathrm{C}_{\text {quat }}\right), 169.65\left(\mathrm{C}_{\text {quat }}\right), 171.00\left(\mathrm{C}_{\text {quat }}\right) . \mathrm{MS}$ [ESI, $\left.\mathrm{H}_{2} \mathrm{O} / \mathrm{AcN}\right]: \mathrm{m} / \mathrm{z}$ $(\%)=320.1[\mathrm{M}+](100)$ 

(rac-4n)

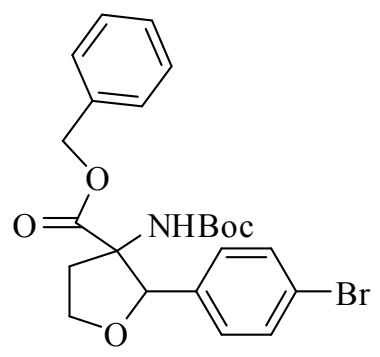

Yield $=20 \%$, using $\mathrm{KOH}$ as a base; $25 \%$, using $\mathrm{CsOH}$ as a base; $R_{\mathrm{f}}=0.14$ (diethyl ether : $\mathrm{PE}$ $=3: 17)$, m.p. $=137-138^{\circ} \mathrm{C}$.

${ }^{1} \mathrm{H}$ NMR $\left(300 \mathrm{MHz}, \mathrm{CDCl}_{3}\right) \delta=1.43(\mathrm{~s}, 9 \mathrm{H}), 2.62(\mathrm{~m}, 1 \mathrm{H},-\mathrm{CH} H-), 2.82$ (m, 1H, -CHH-), 4.20 (m, 1H, -OCHH-), 4.37(m, 1H, -OCHH-), 4.72 (s, 2H), 4.96 (bs, 1H), 5.54 (bs, 1H,), $7.13(\mathrm{~m}, 4 \mathrm{H}), 7.33(\mathrm{~m}, 5 \mathrm{H}) .-{ }^{13} \mathrm{C} \mathrm{NMR}\left(75.5 \mathrm{MHz}, \mathrm{CDCl}_{3}\right) \delta=28.32(+), 35.20(-), 67.58(-)$, $67.97(-), 70.04(+), 80.50\left(\mathrm{C}_{\text {quat }}\right), 85.17$ (Cquat), $122.29\left(\mathrm{C}_{\text {quat }}\right), 127.69(+), 128.46(+)$, $128.50(+), 128.54(+), 131.23(+), 134.72\left(\mathrm{C}_{\text {quat }}\right), 135.99\left(\mathrm{C}_{\text {quat }}\right), 154.51\left(\mathrm{C}_{\text {quat }}\right), 170.79$ $\left(\mathrm{C}_{\text {quat }}\right)$. - MS [ESI; $\left.\mathrm{CH}_{2} \mathrm{Cl}_{2} / \mathrm{MeOH}+10 \mathrm{mmol} \mathrm{NH}_{4} \mathrm{OAc}\right] .=476.1,478.1\left[\mathrm{MH}^{+}\right]$(50), 493.2, $495.2\left(\mathrm{M}+\mathrm{NH}_{4}{ }^{+}\right)-\mathrm{IR}(\mathrm{KBr}): \widetilde{v} \mathrm{~cm}^{-1}=3348,3303,3061,3029,2978,2941,2887,2867$, 2800, 2199, 1668, 1591, 1517, 1452. Anal. calcd. For $\mathrm{C}_{23} \mathrm{H}_{26} \mathrm{BrNO}_{5}$ (476.19): C 57.99, $\mathrm{H}$ 5.50, N 2.94, found C 57.95, H 5.72, N 2.93 .

tert-Butyl 3-(tert-butoxycarbonylamino)-2-(4-morpholinophenyl)tetrahydrofuran-3 carboxylate (rac-16b)

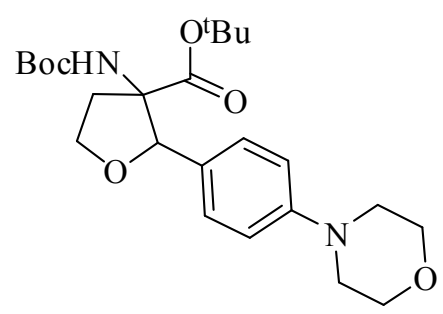

The compound was prepared by using the same procedure as described for rac-16a. The isolated yield was $35 \%$.

${ }^{1} \mathrm{H}$ NMR $\left(300 \mathrm{MHz}, \mathrm{CDCl}_{3}\right): \delta=1.14(\mathrm{~s}, 9 \mathrm{H}), 1.50(\mathrm{~s}, 9 \mathrm{H}), 2.52--2.60(\mathrm{~m},-\mathrm{CH} H-, 1 \mathrm{H})$, 2.70-2.80 (m, -CHH-, 1H), $3.10(\mathrm{~m}, 4 \mathrm{H})$ 3.80-3.90 (m, 4H), 4.09-4.18 (m, -OCHH-, 1H), 
4.27-4.38 (m, -OCHH-, 1H), 4.90 (bs, 1H), 5.50 (bs, 1H), 6.80 (d, J=8.42 Hz, 2H), 7.20 (d, $J=8.42 \mathrm{~Hz}, 2 \mathrm{H}) .-{ }^{13} \mathrm{C} \mathrm{NMR}\left(75.5 \mathrm{MHz}, \mathrm{CDCl}_{3}\right): \delta=27.51(+), 28.40(+), 35.94(-), 49.59(-$ ), $66.79(-), 67.74(-), 67.79(+), 69.80(+), 81.95\left(\mathrm{C}_{\text {quat }}\right), 82.50\left(\mathrm{C}_{\text {quat }}\right), 84.72\left(\mathrm{C}_{\text {quat }}\right), 117.73$ $(+), 126.72\left(\mathrm{C}_{\text {quat }}\right), 127.66(+), 131.95(+), 140.16\left(\mathrm{C}_{\text {quat }}\right), 144.47(+), 154.3\left(\mathrm{C}_{\text {quat }}\right), 167.45$ $\left(\mathrm{C}_{\text {quat }}\right), 170.05$ ( $\left.\mathrm{C}_{\text {quat }}\right)$ - - $\mathrm{MS}$ [ESI; $\left.\mathrm{CH}_{2} \mathrm{Cl}_{2} / \mathrm{MeOH}+10 \mathrm{mmol} \mathrm{NH} 4 \mathrm{OAc}\right] .=449.2\left[\mathrm{MH}^{+}\right](100)$. 
Figure S-1. Structure of compound rac-4n in the solid state.

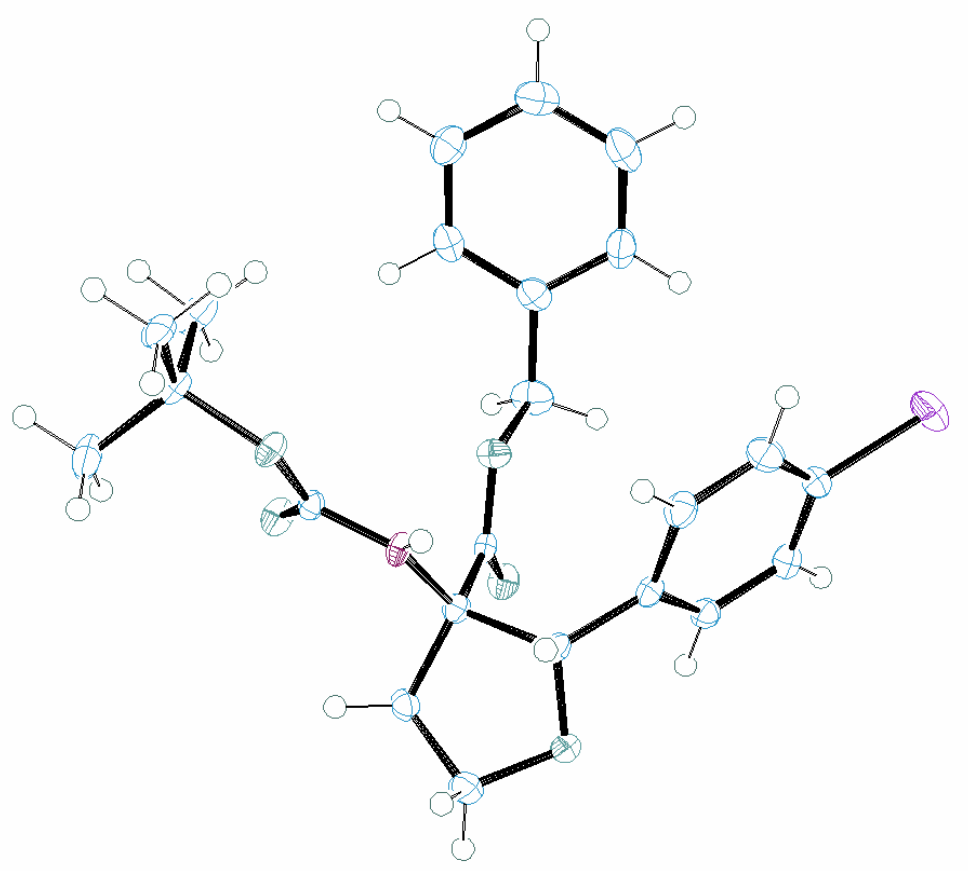

Figure S-2. Structure of compound 7 ( $R, S, S$-isomer) in the solid state.

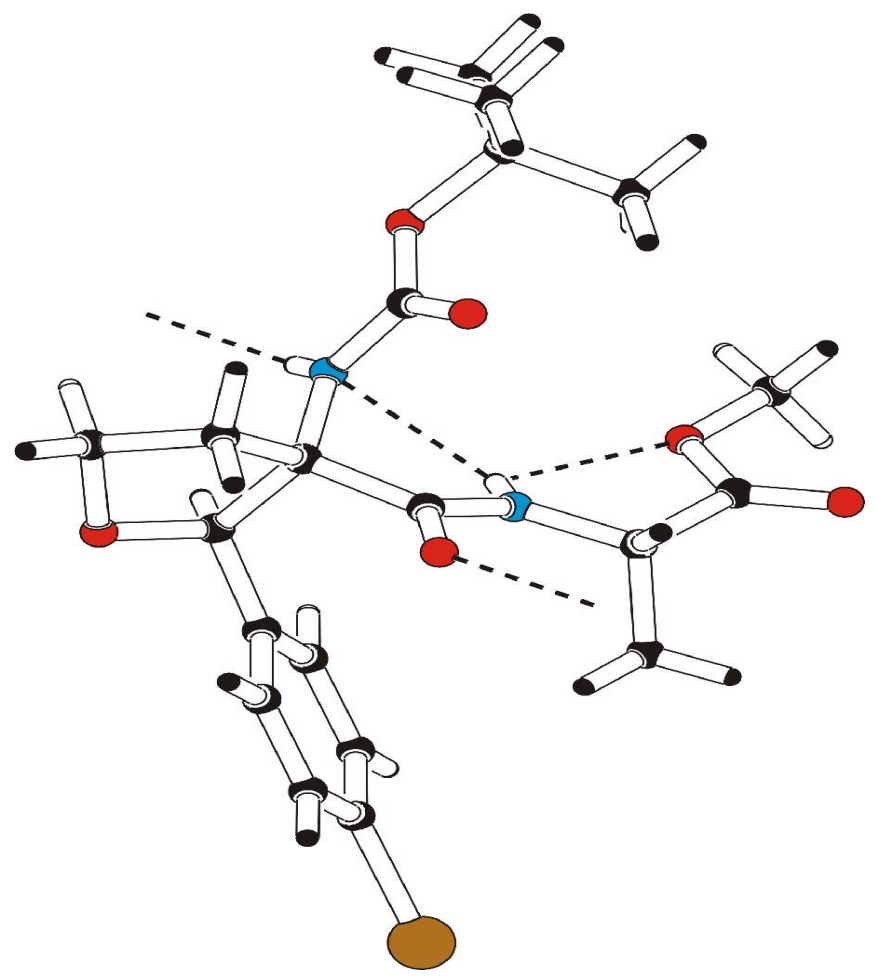


Figure S-3. Structure of compound $9(S, R, S$-isomer) in the solid state

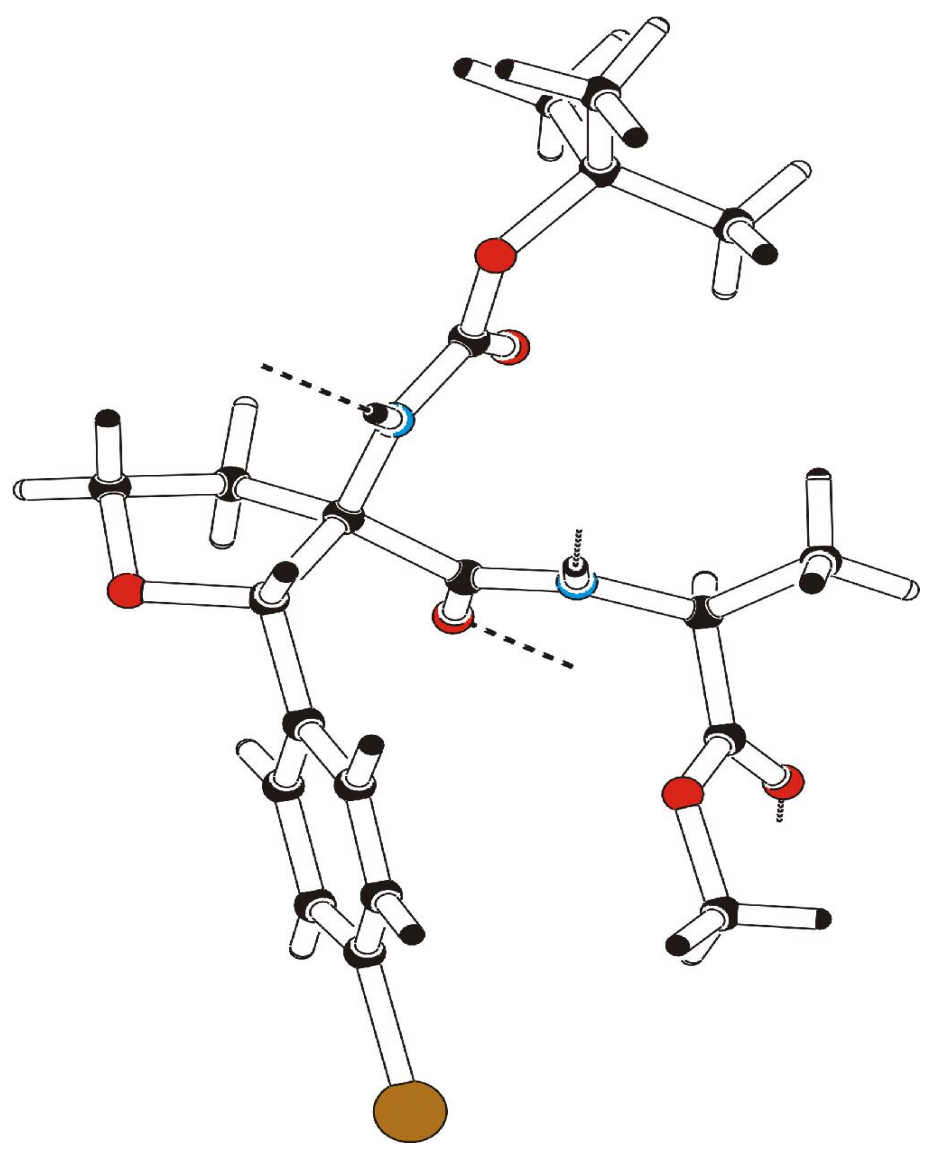

Figure S-4. Structure of compound 12 (S,R,S isomer) in the solid state.

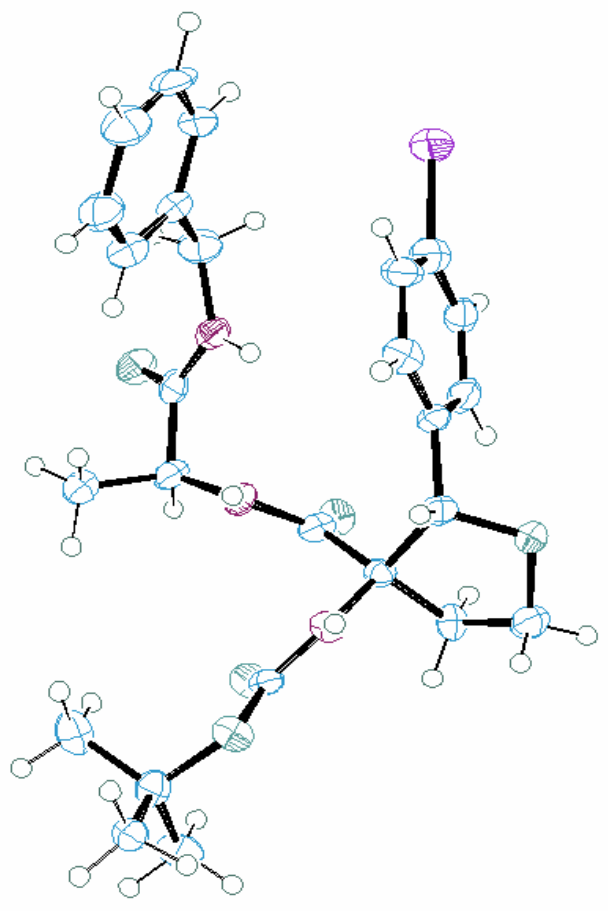


3) Copies of ${ }^{1} \mathrm{H}$ NMR spectra of all new compounds

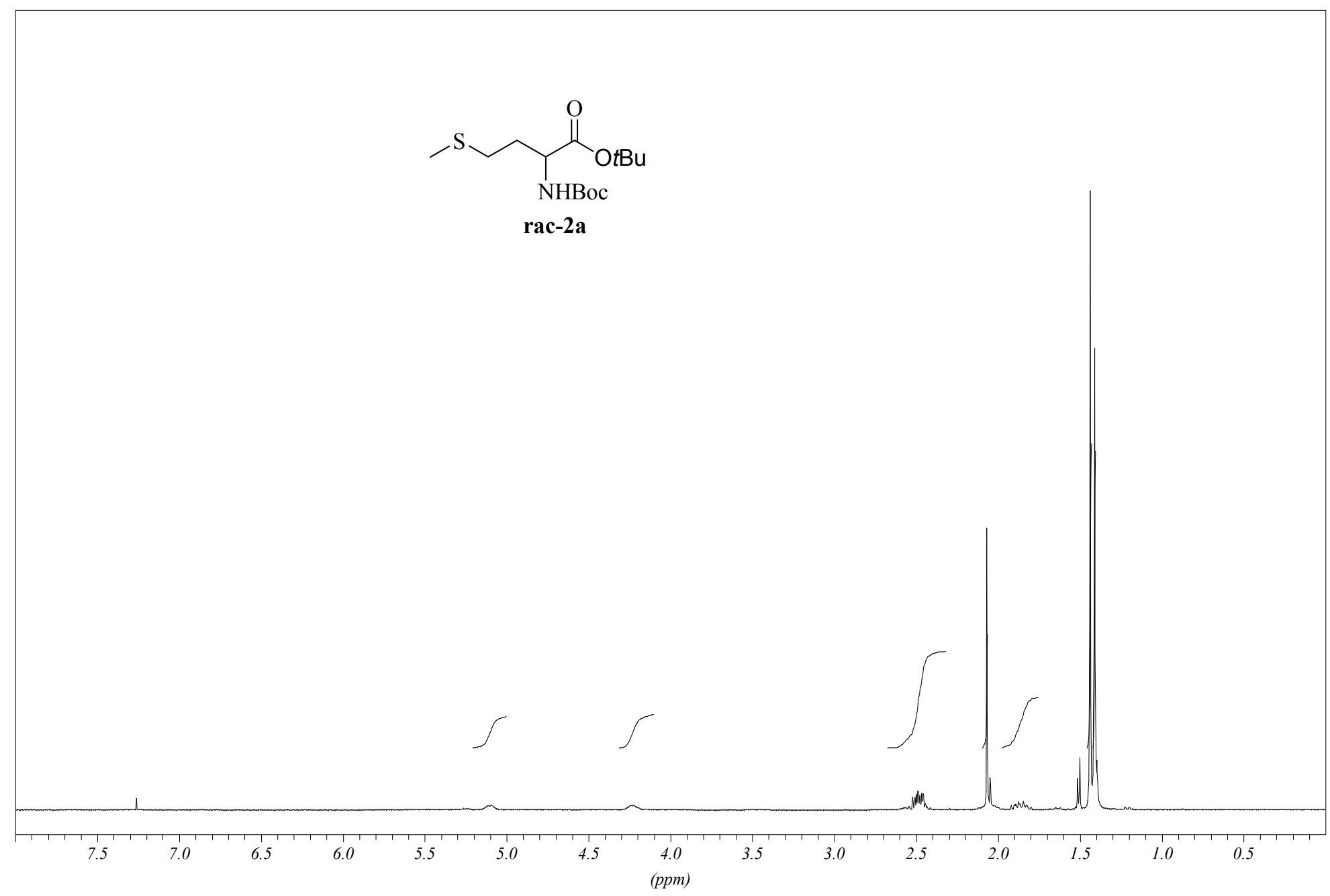




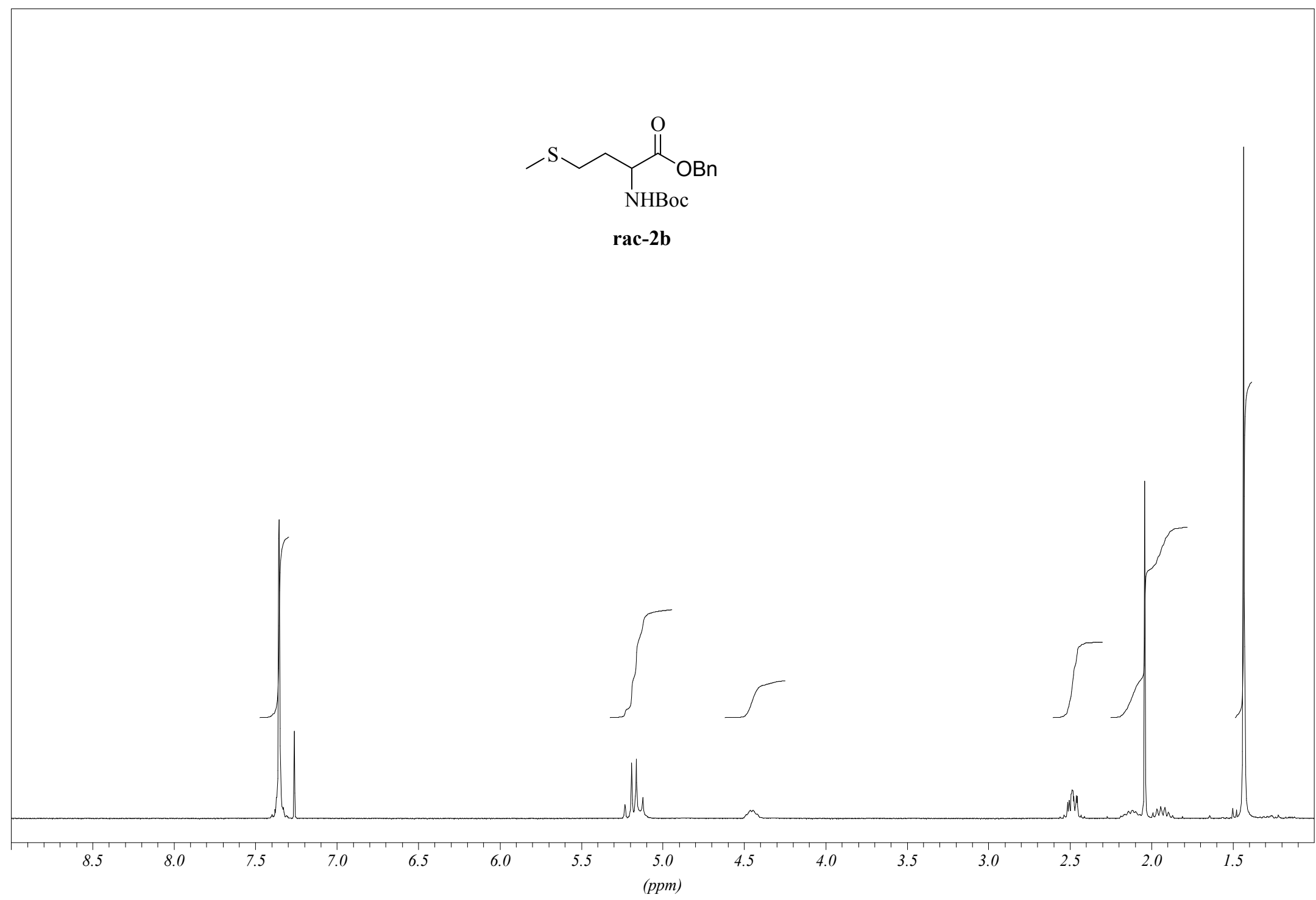




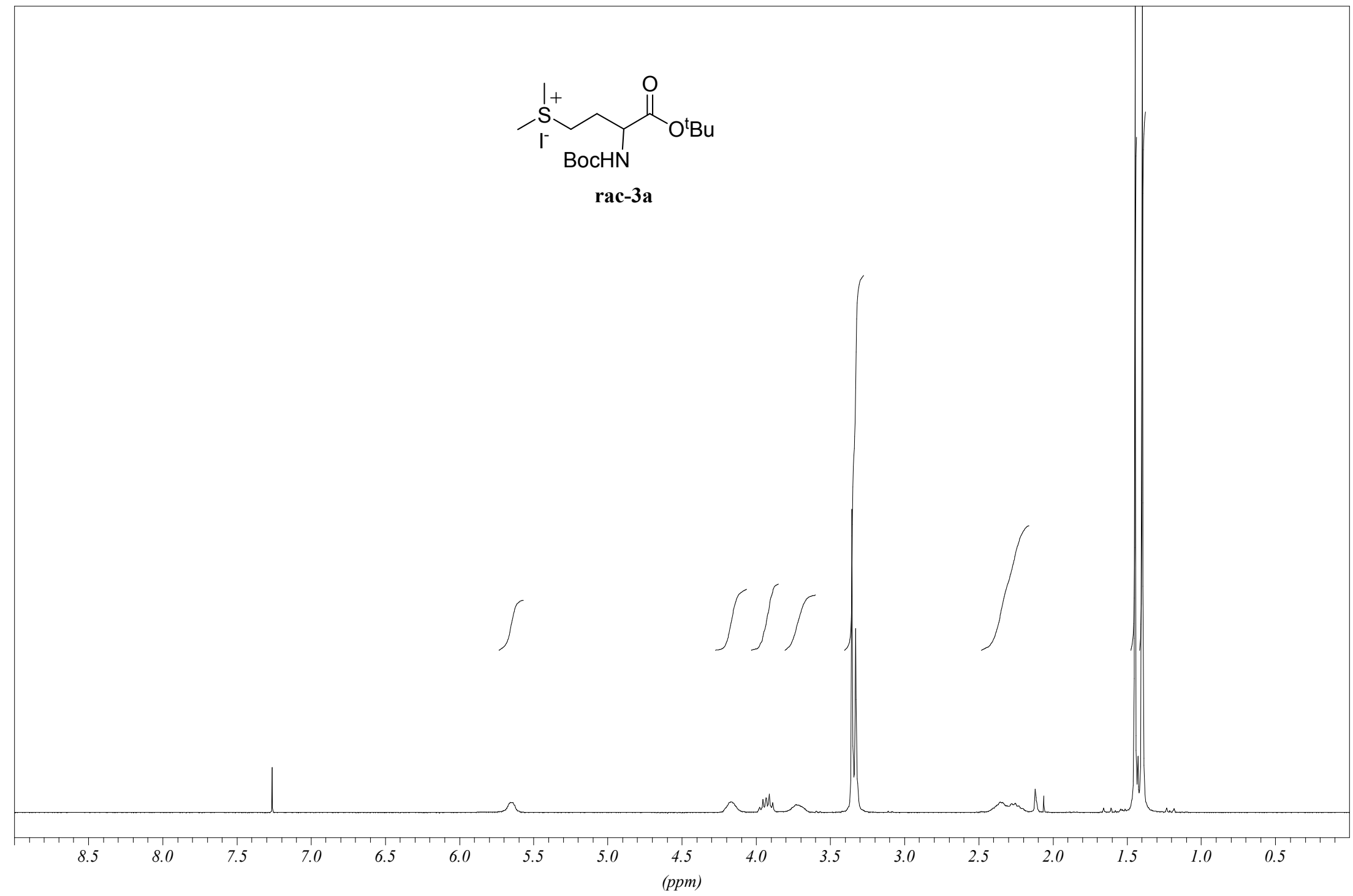

S-15 


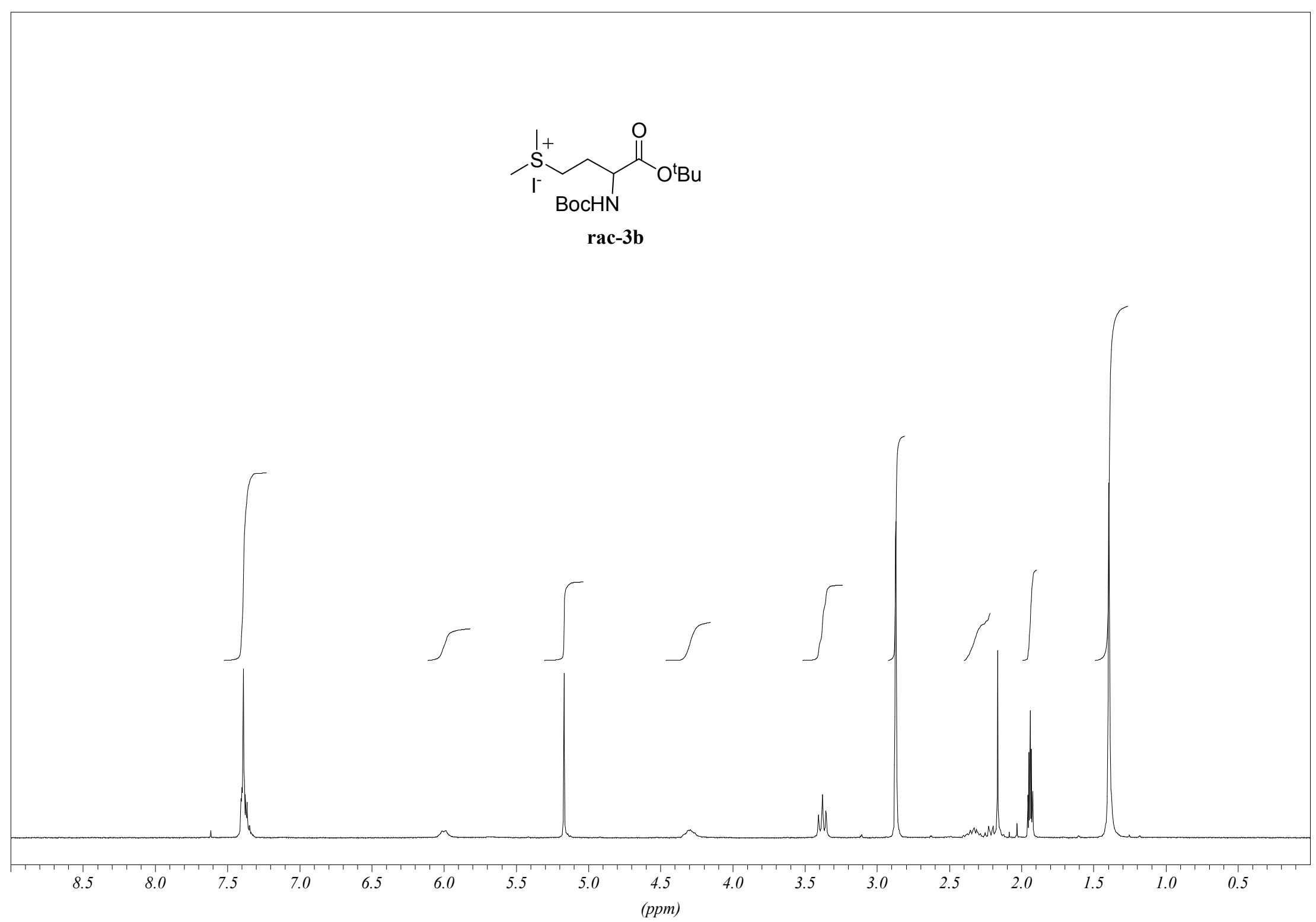

S-16 


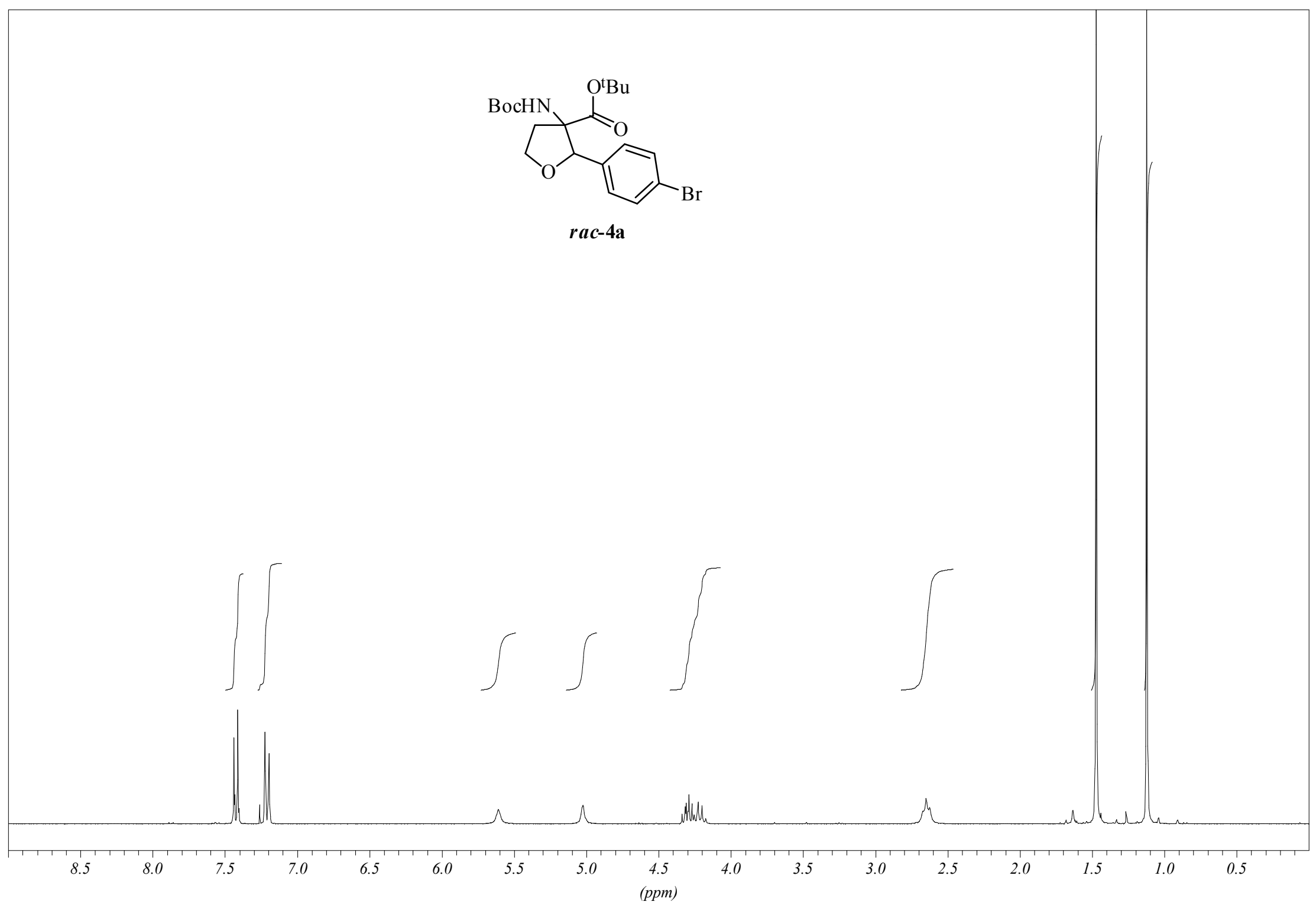




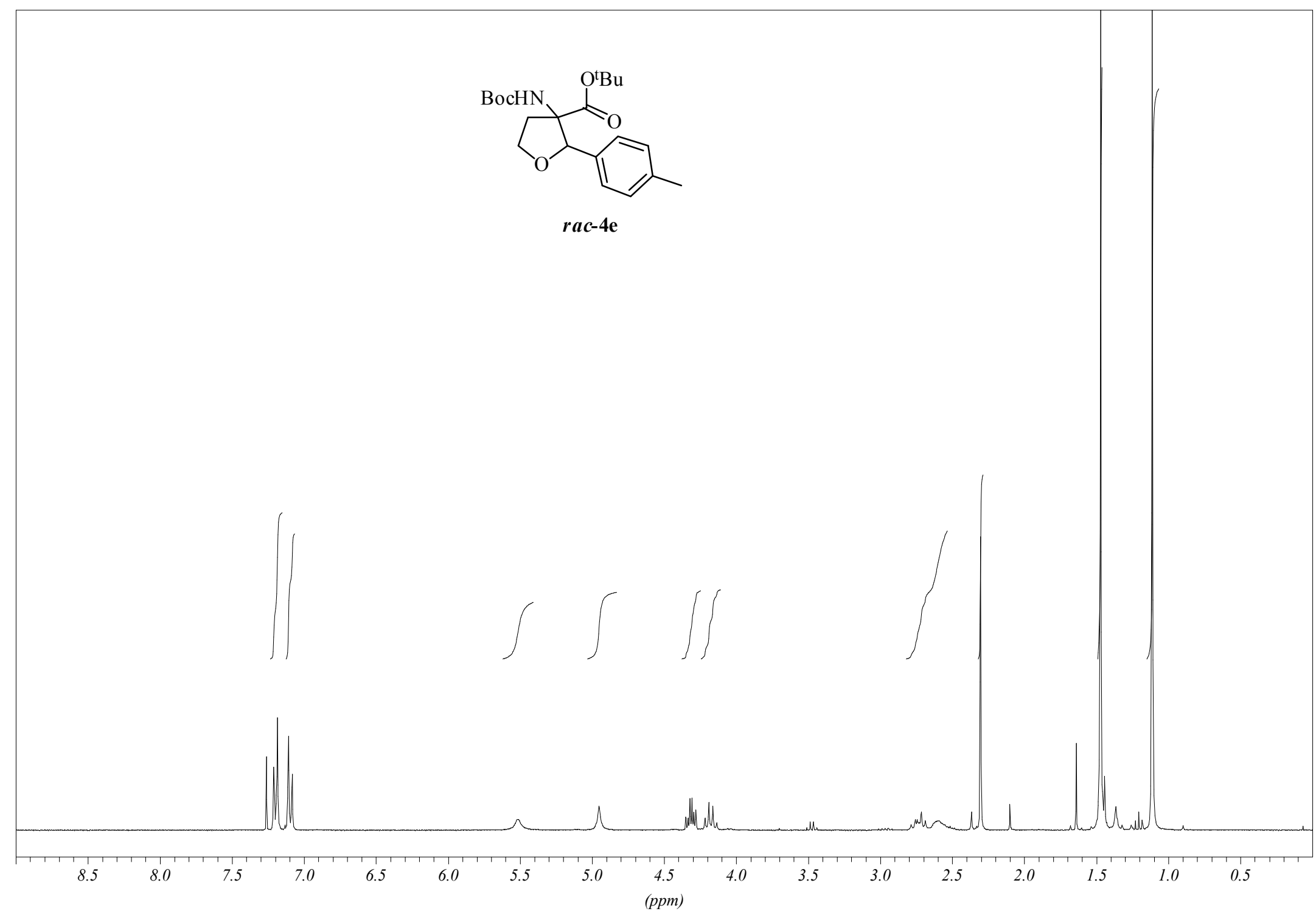

S-18 


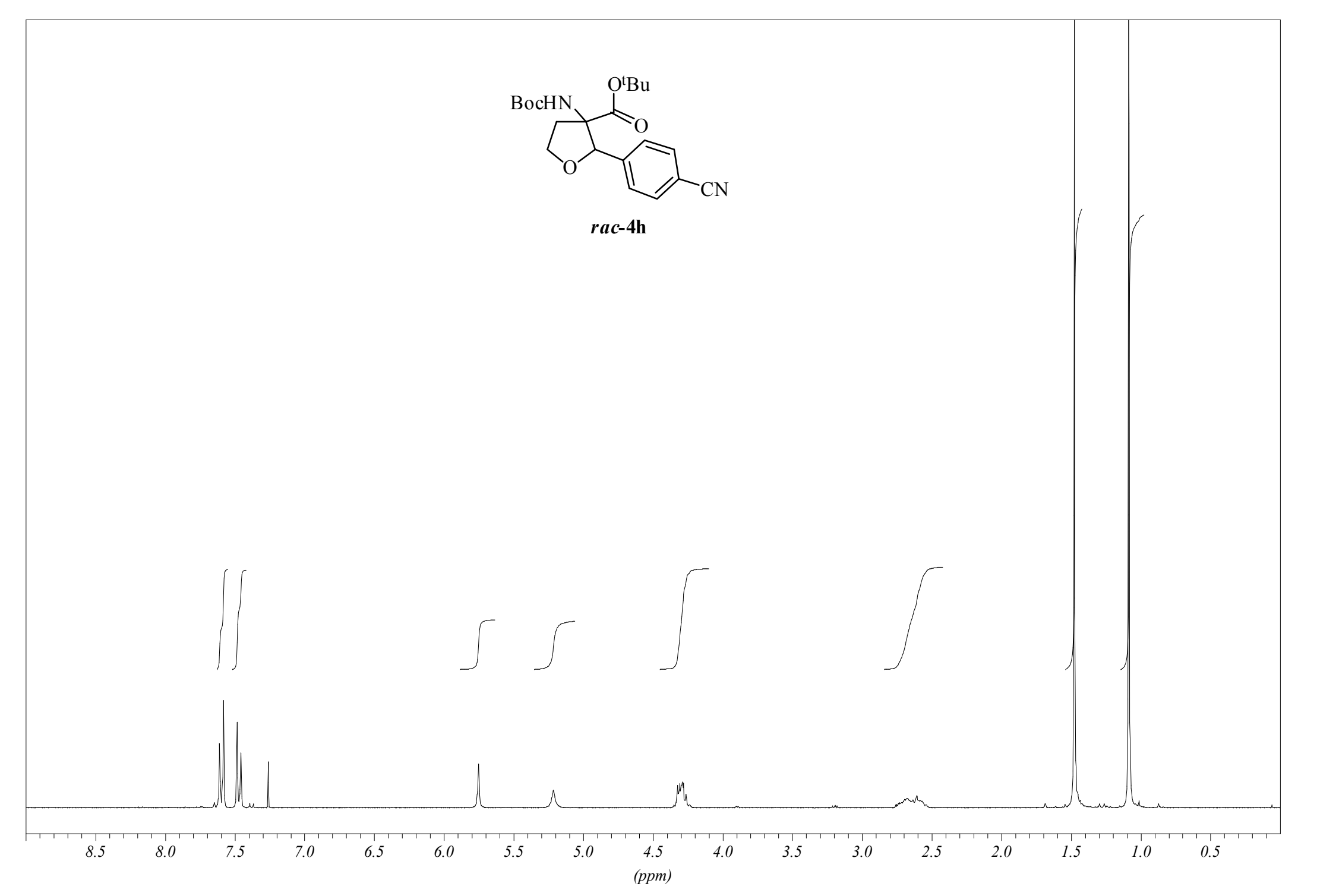




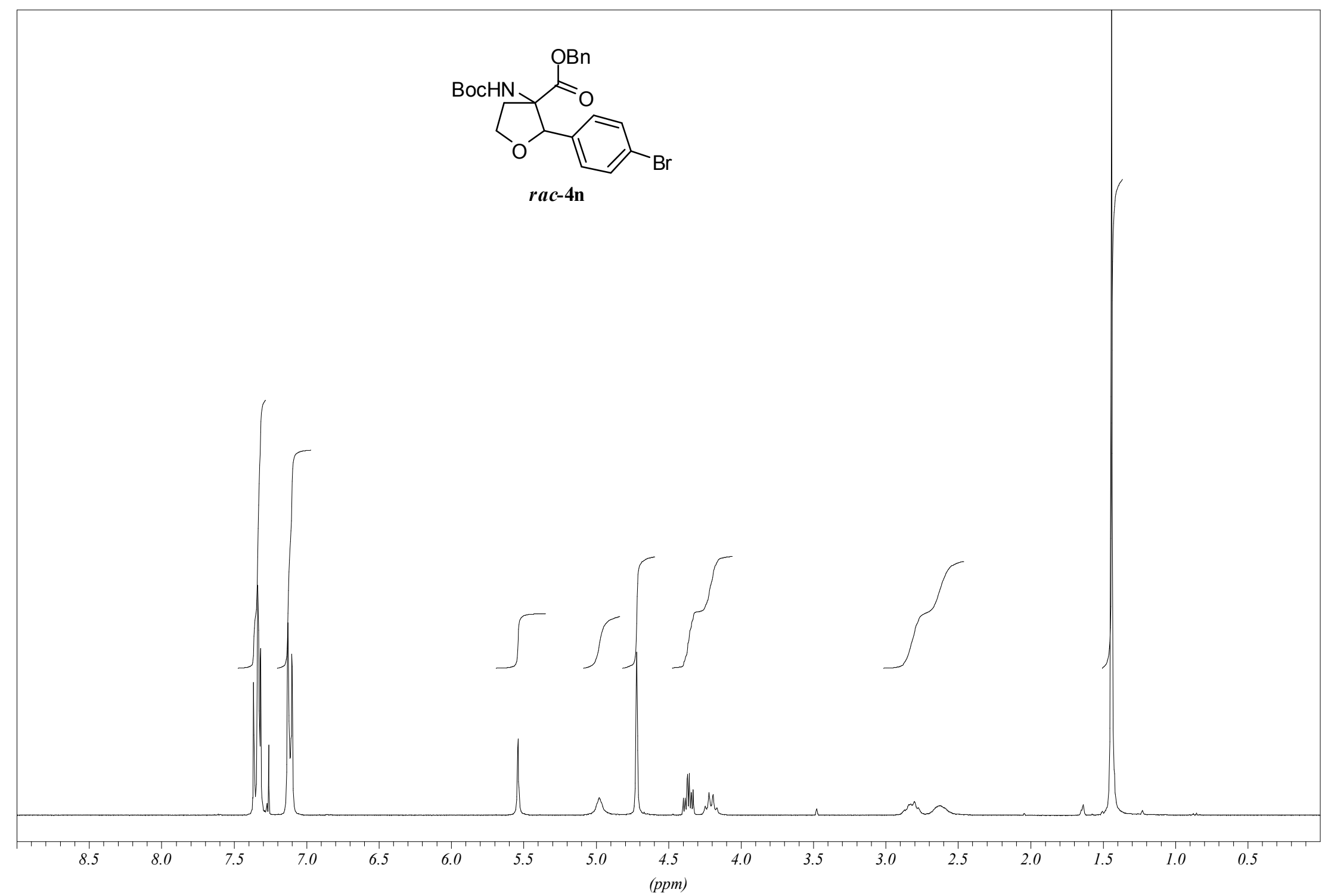




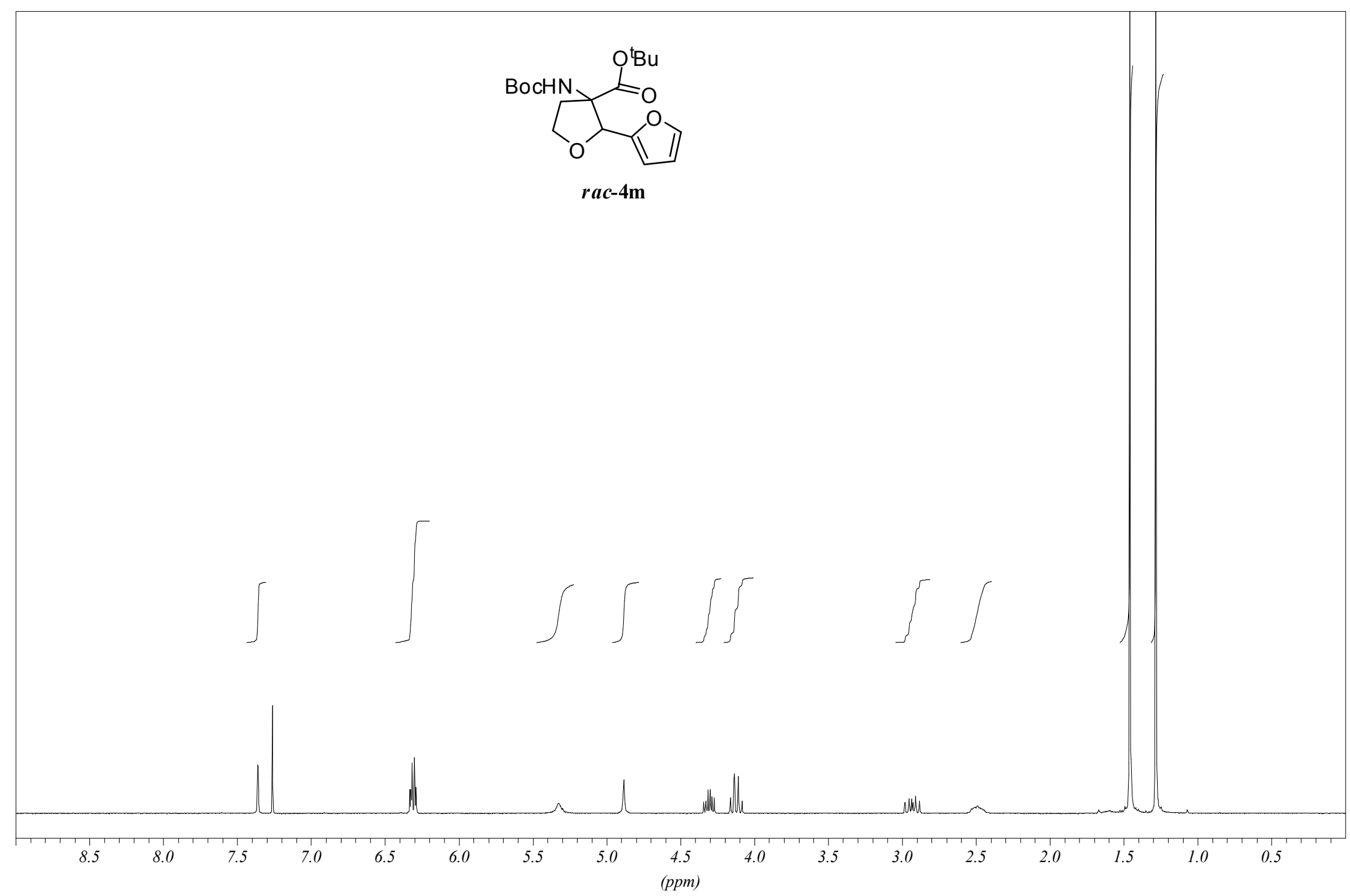




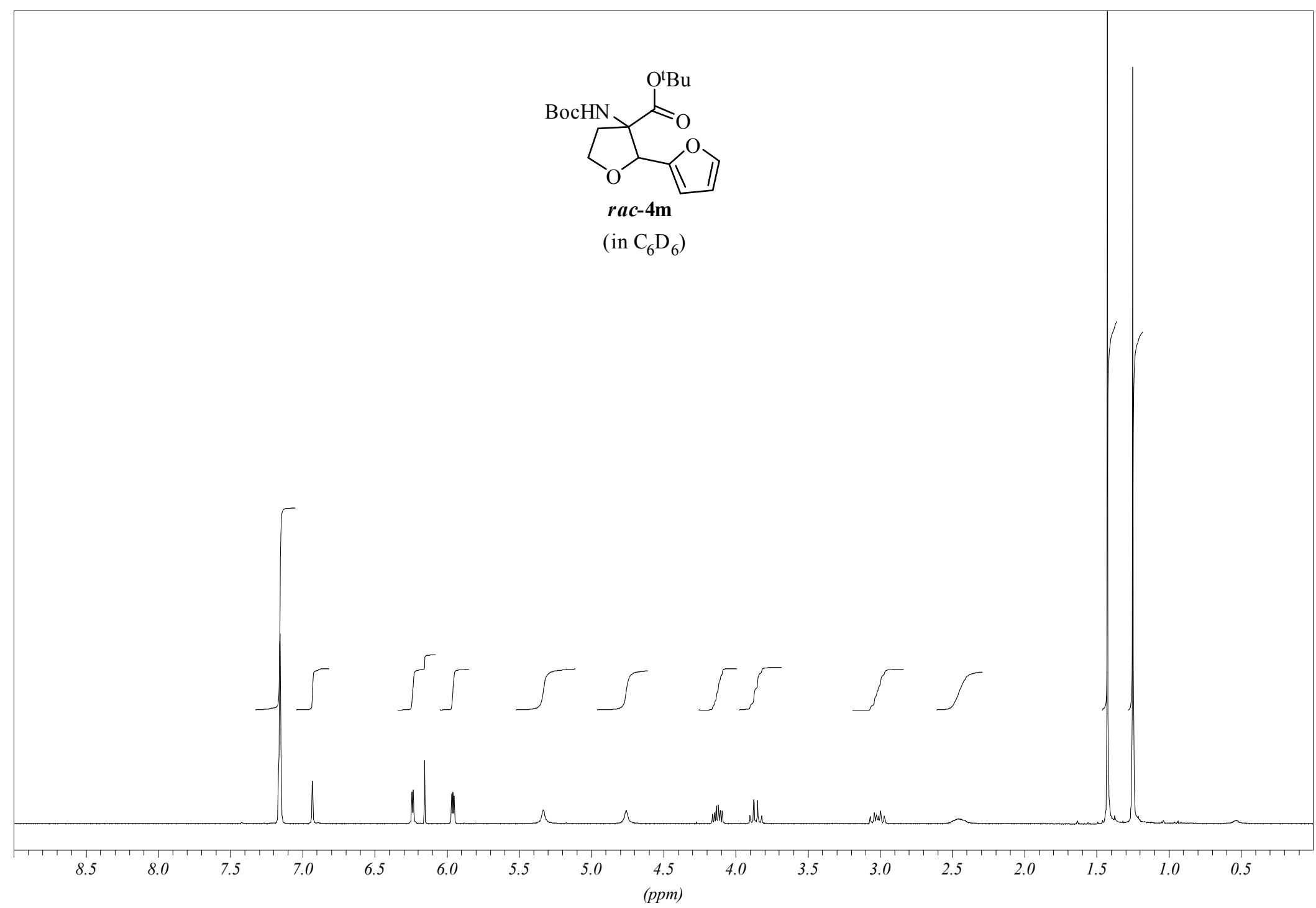




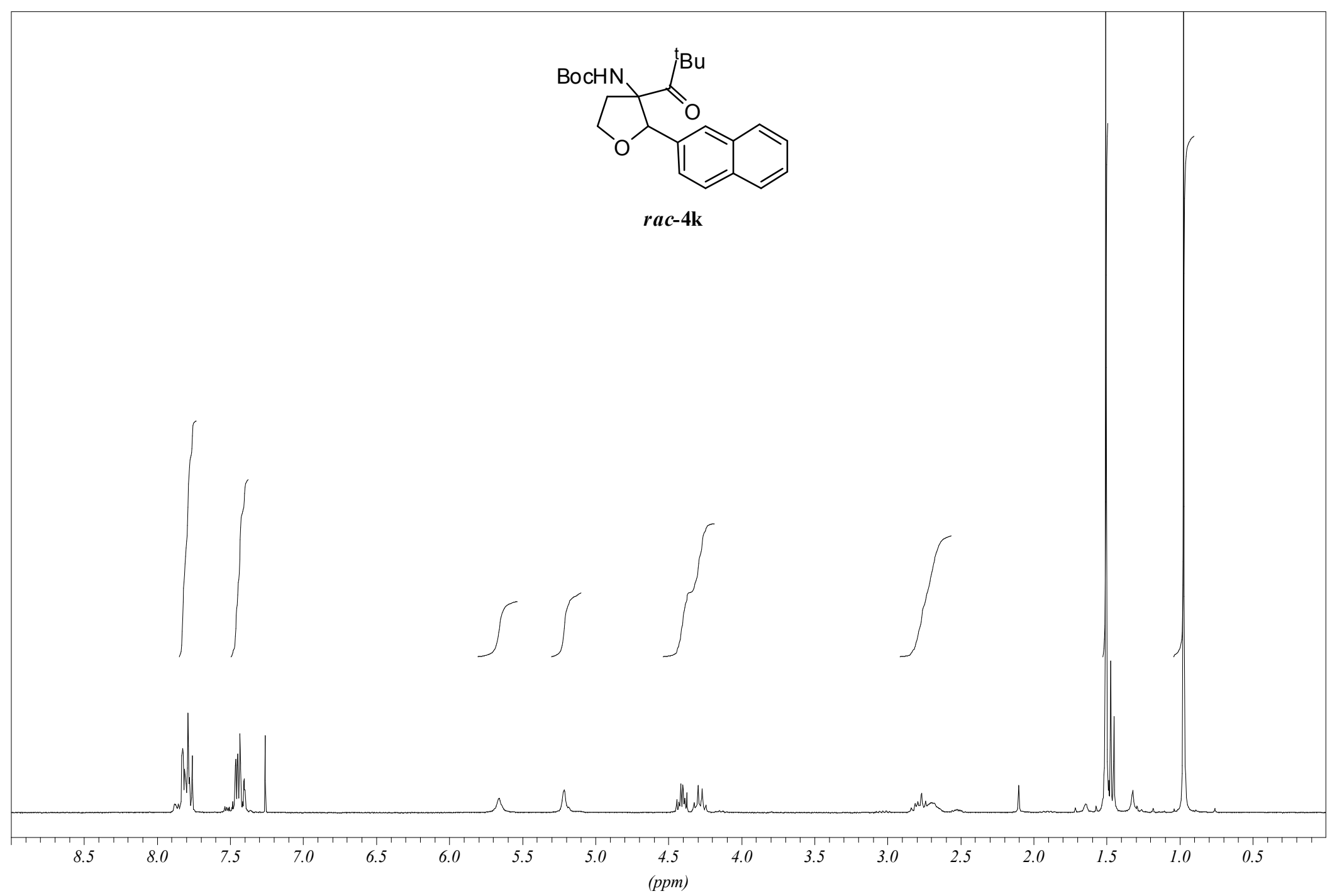




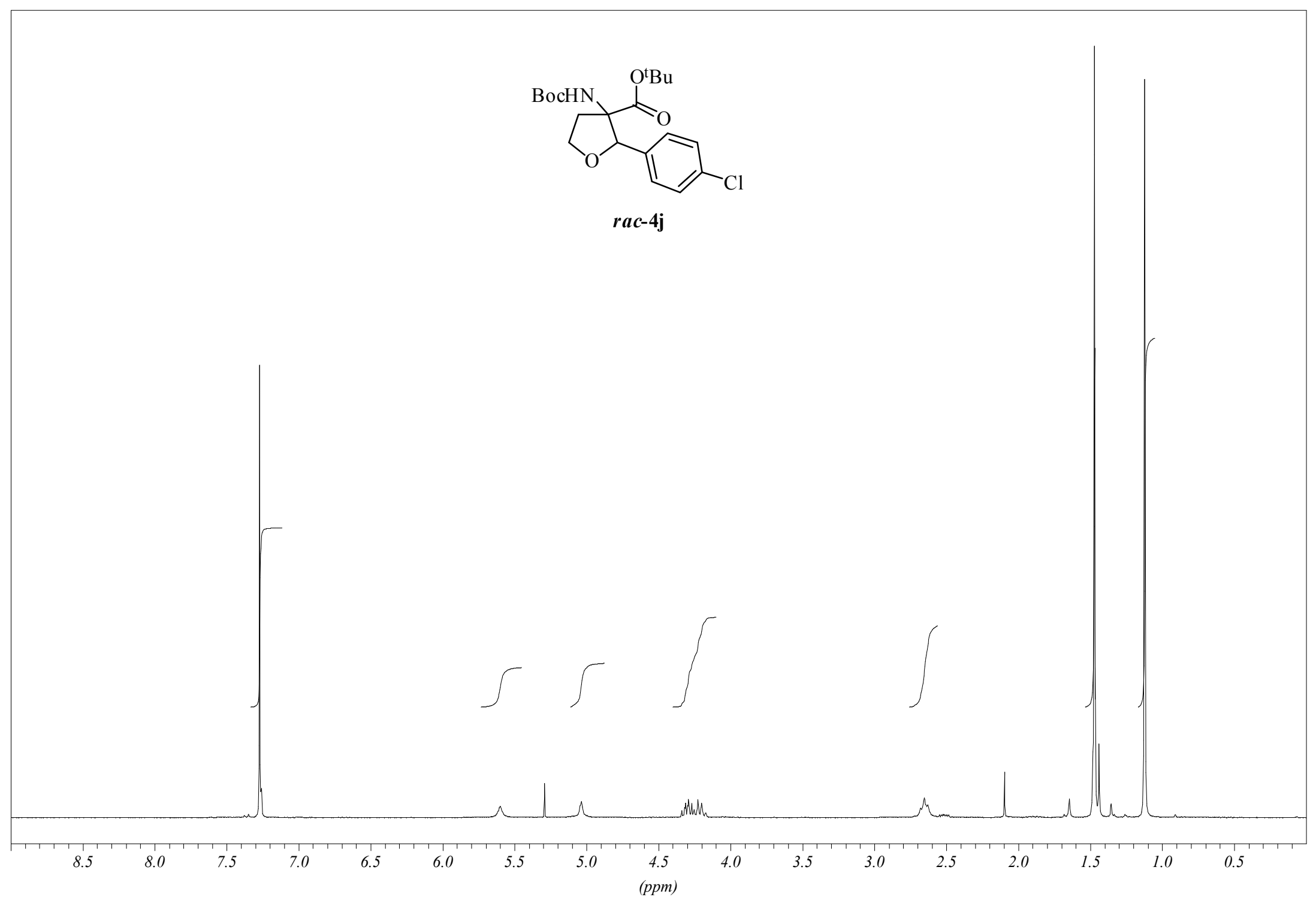




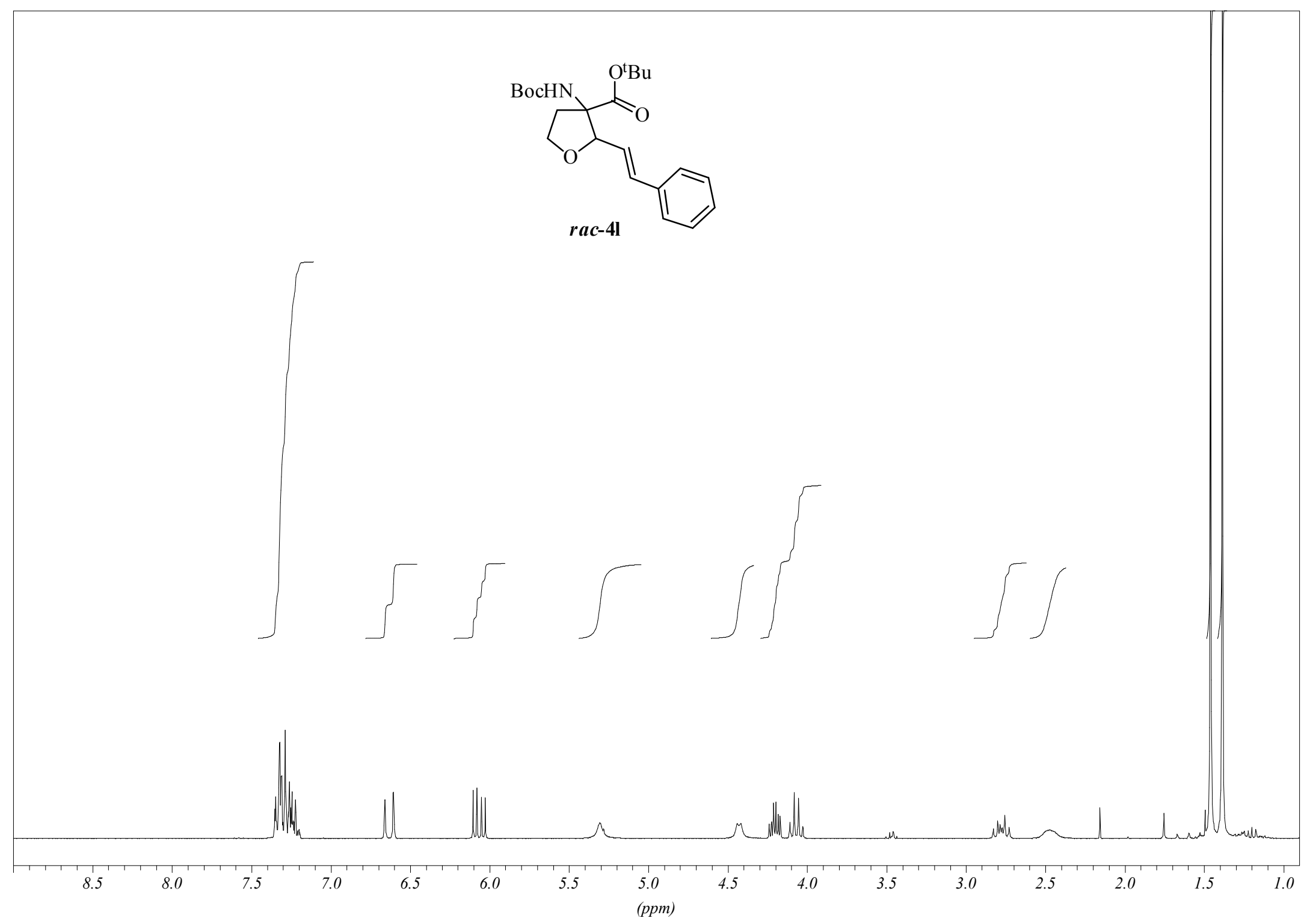




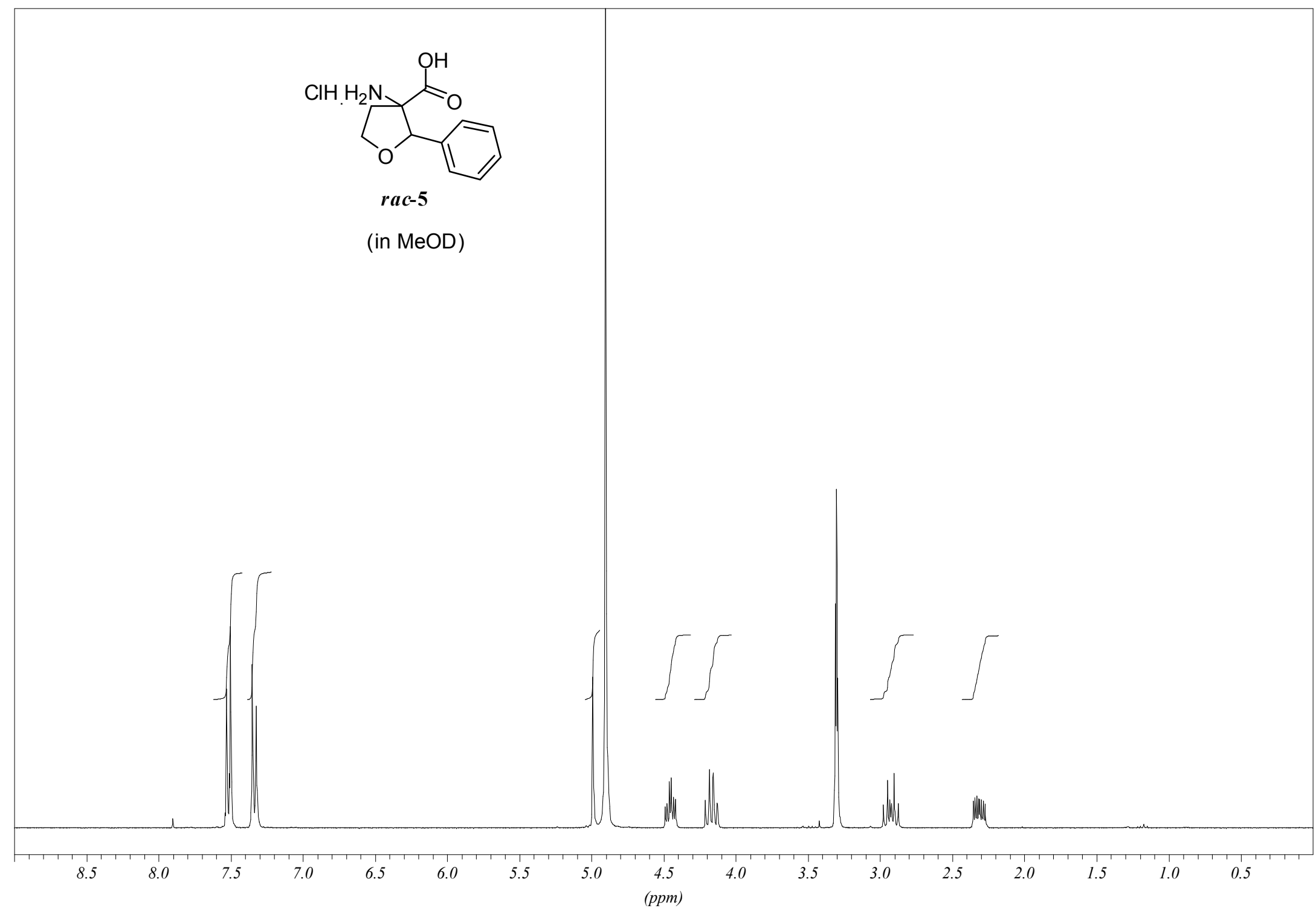

S-26 


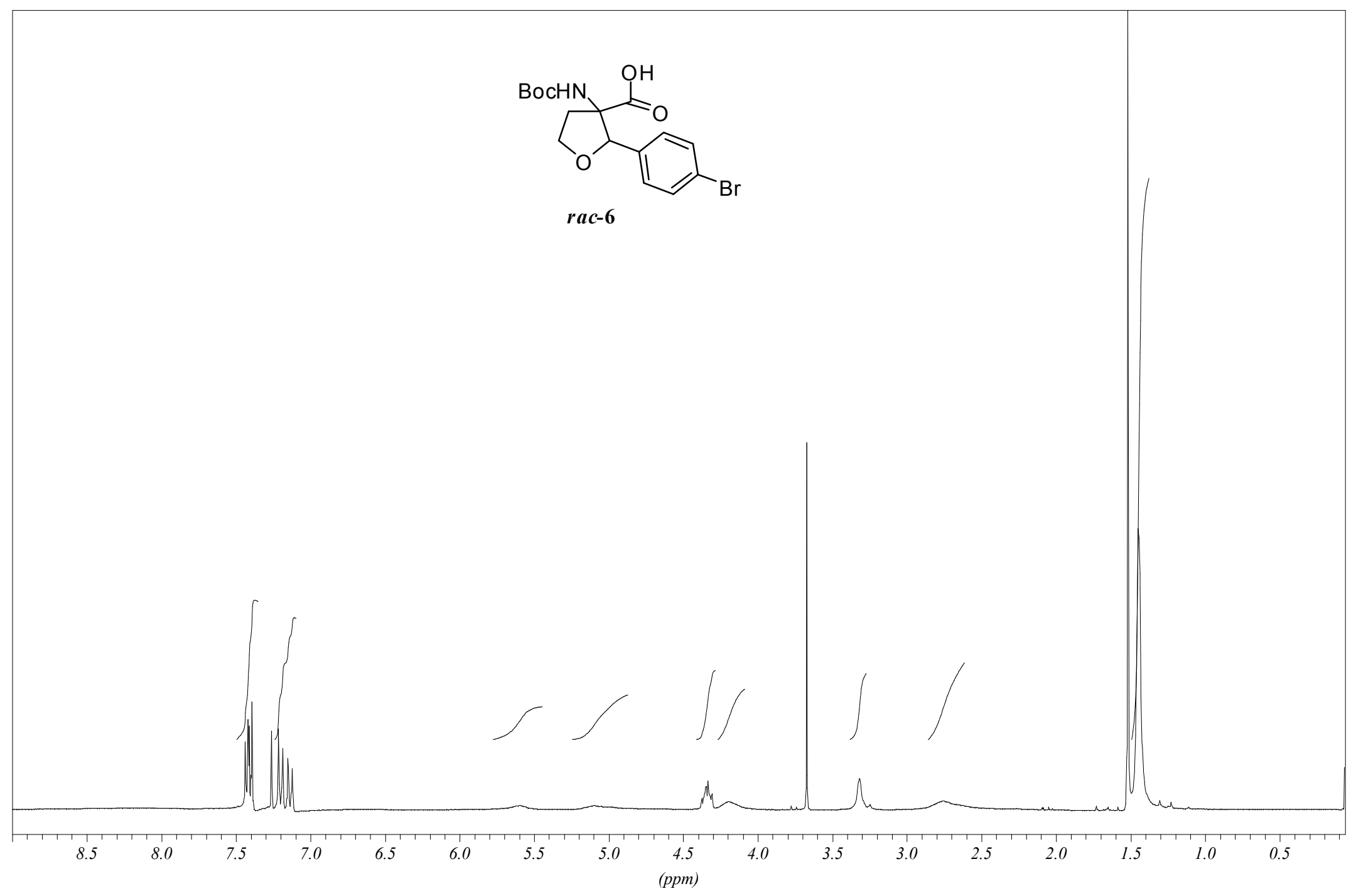




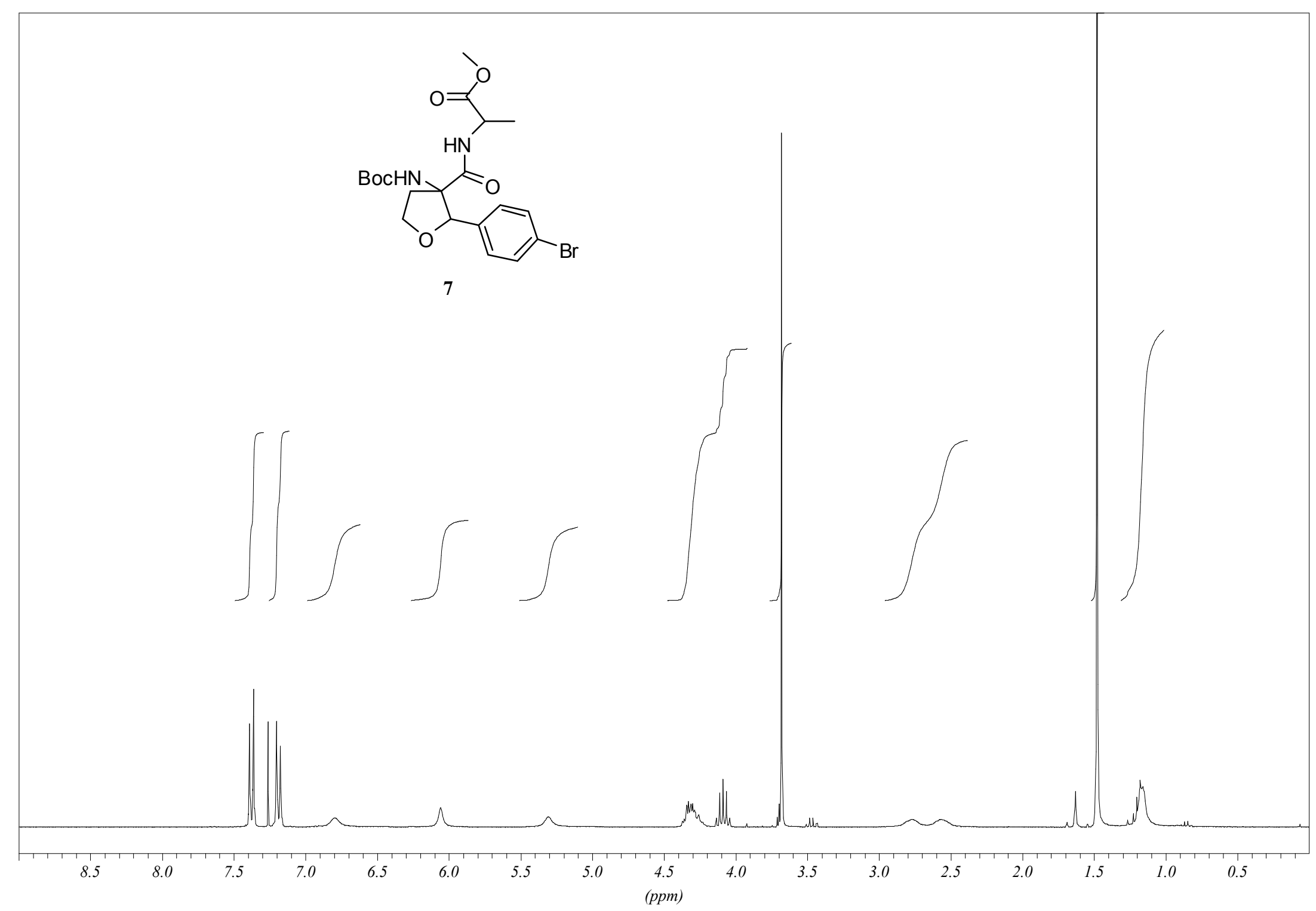




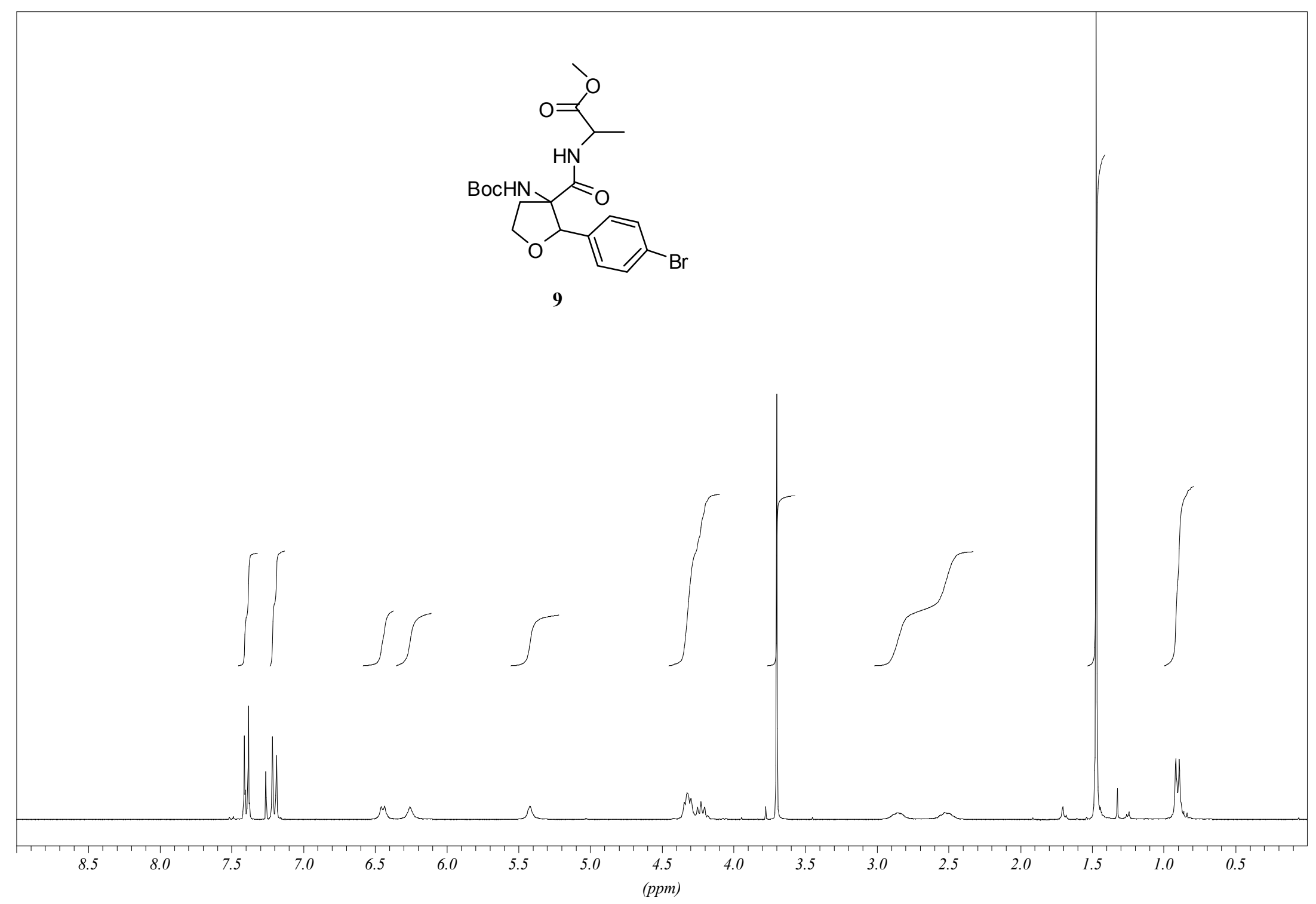

S-29 


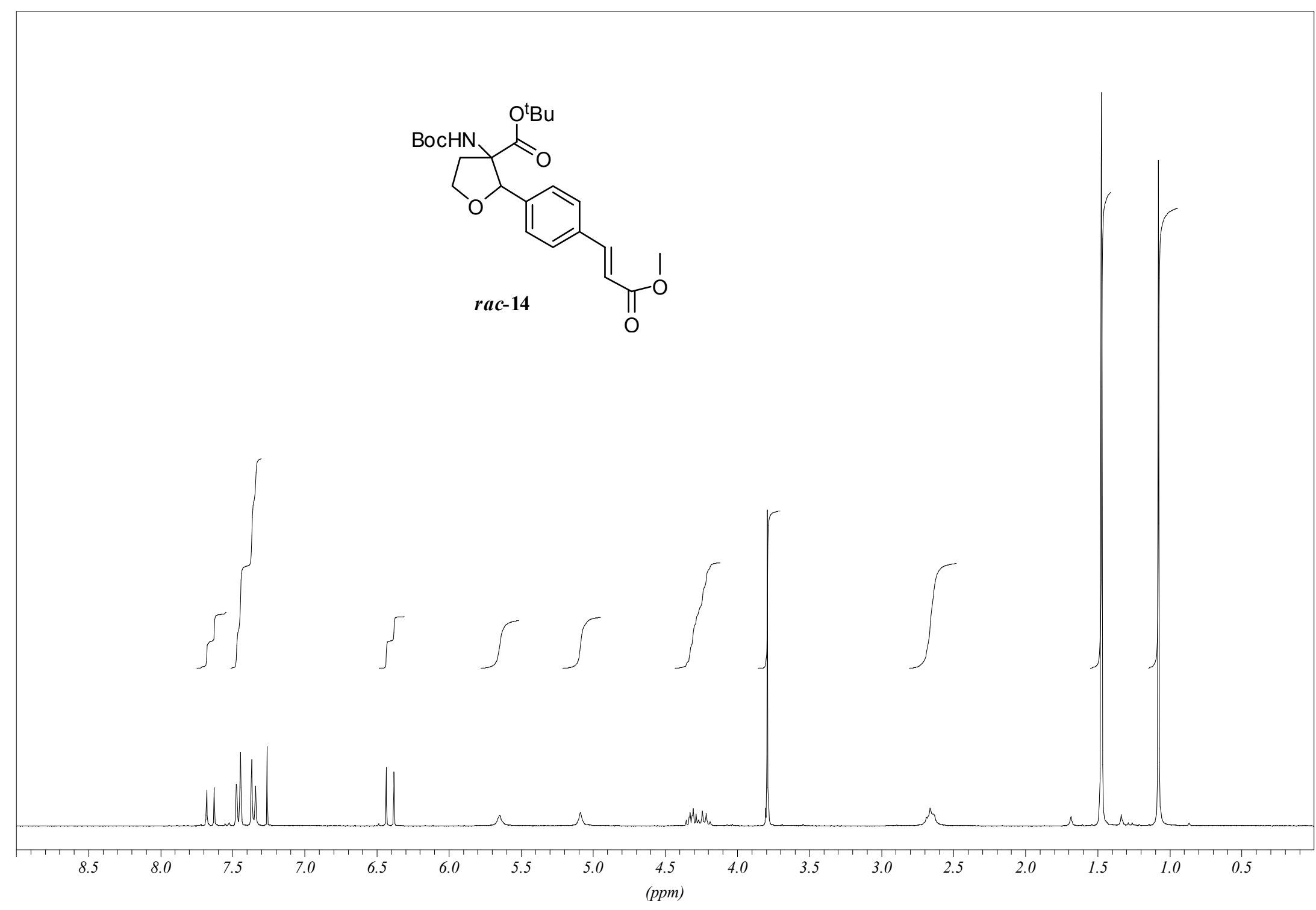




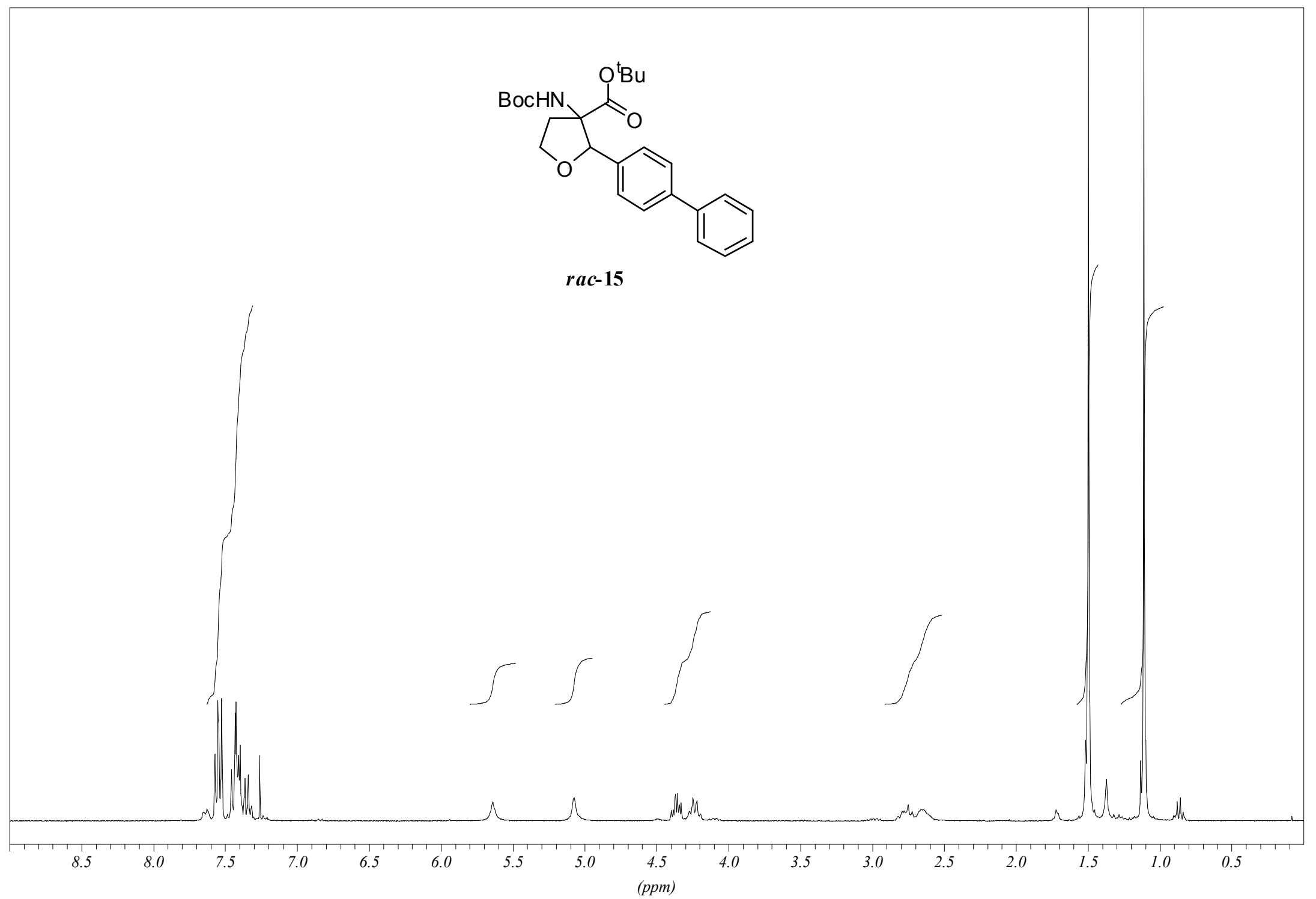

S-31 


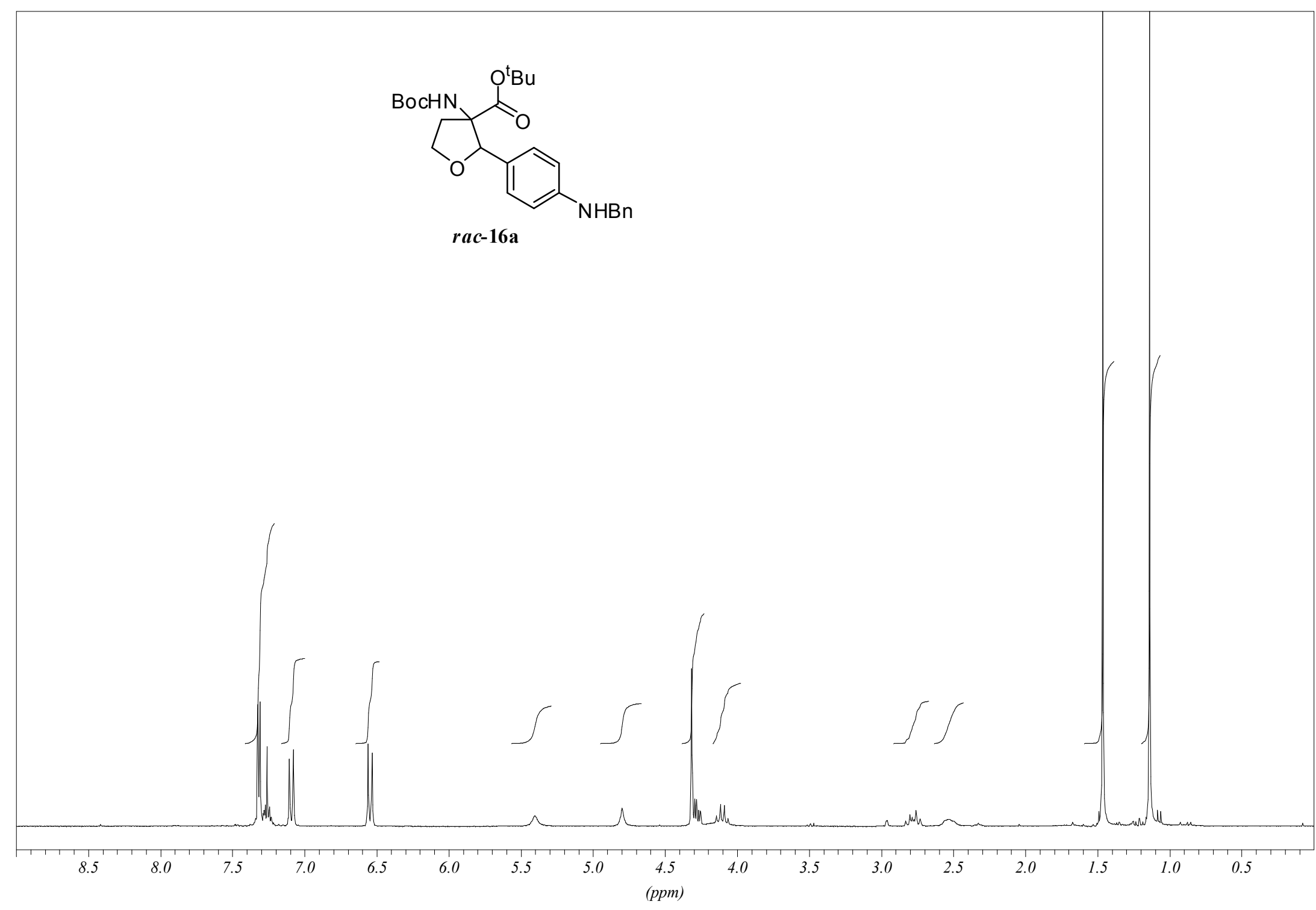




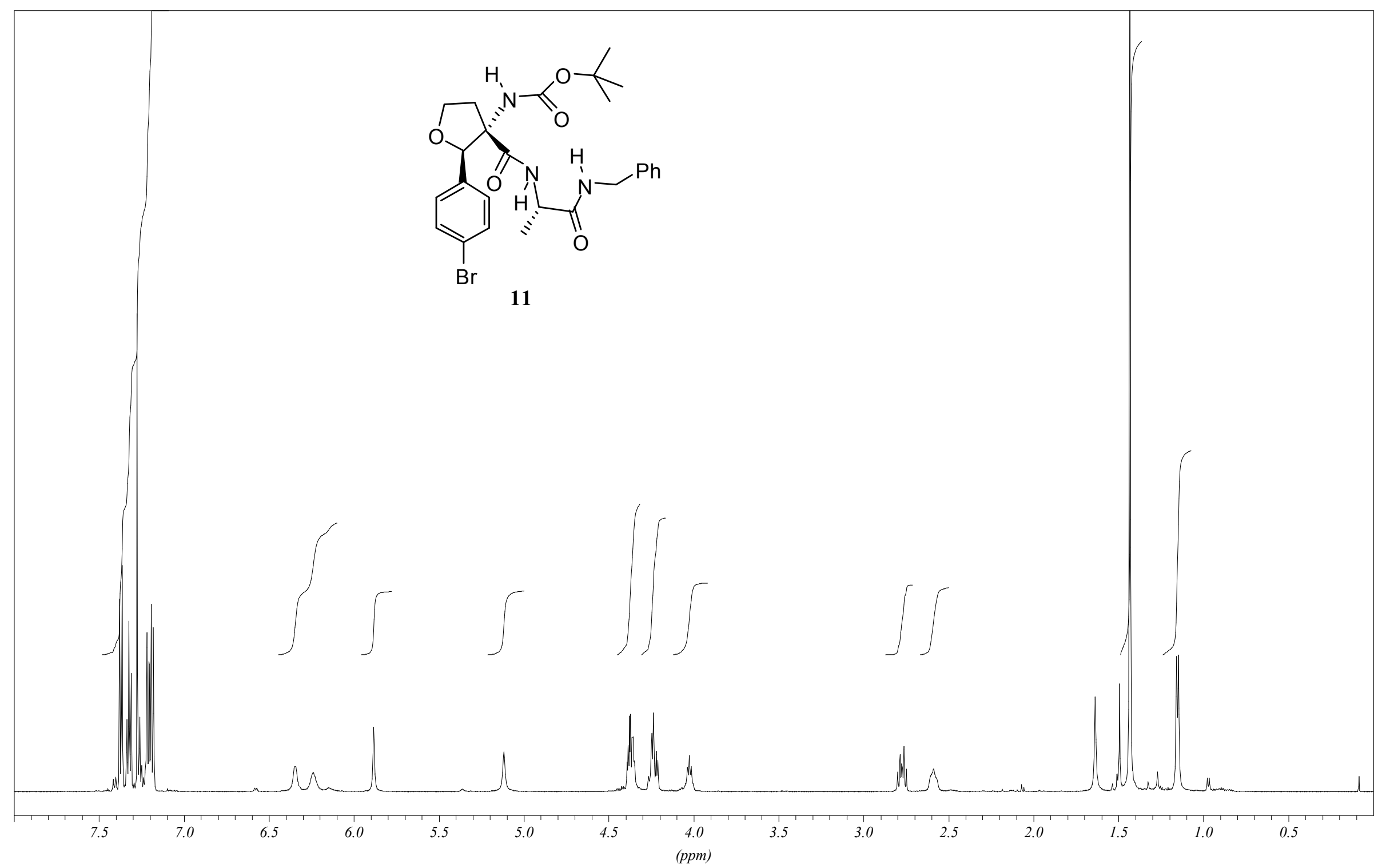




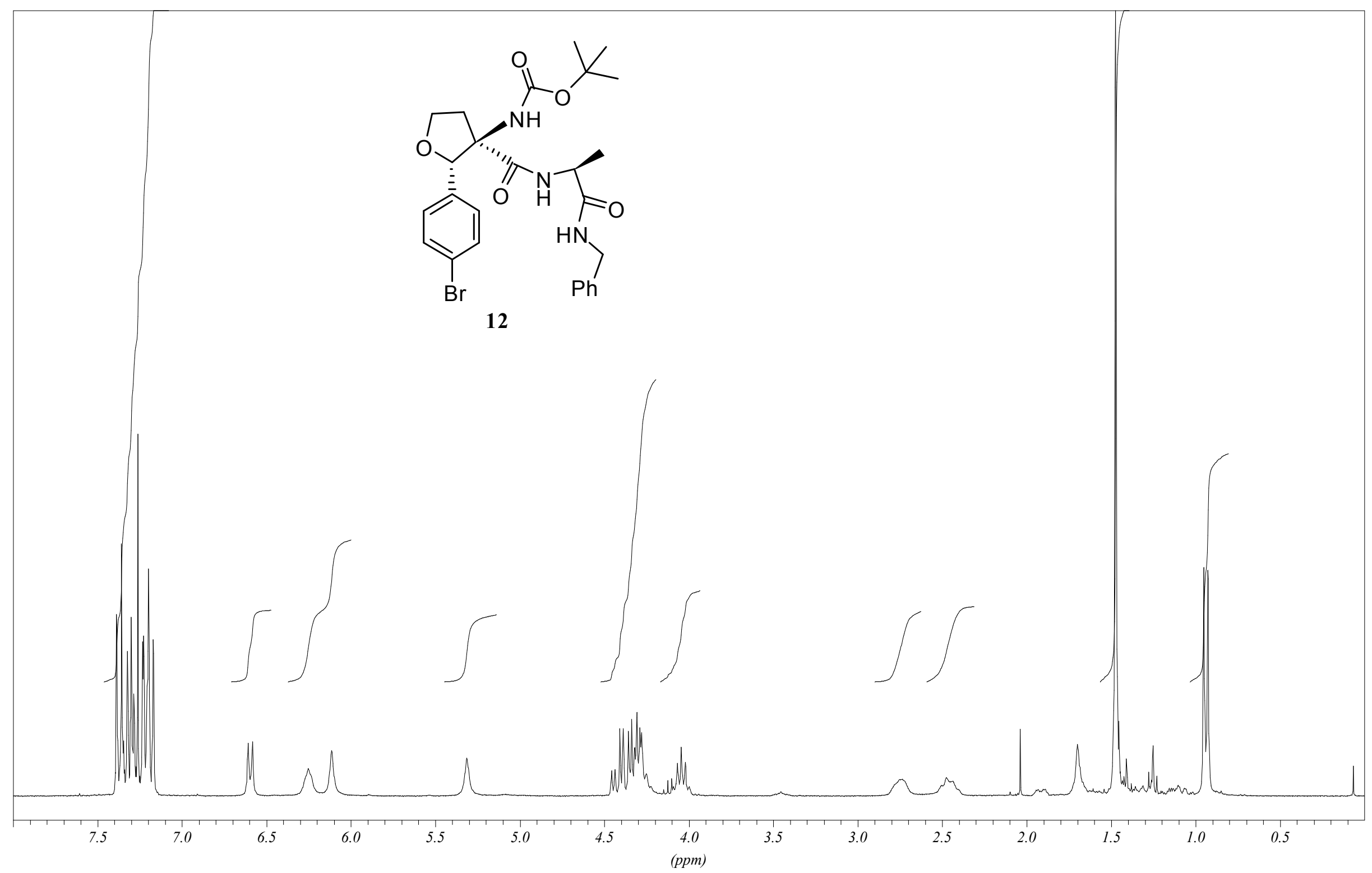

S-34 


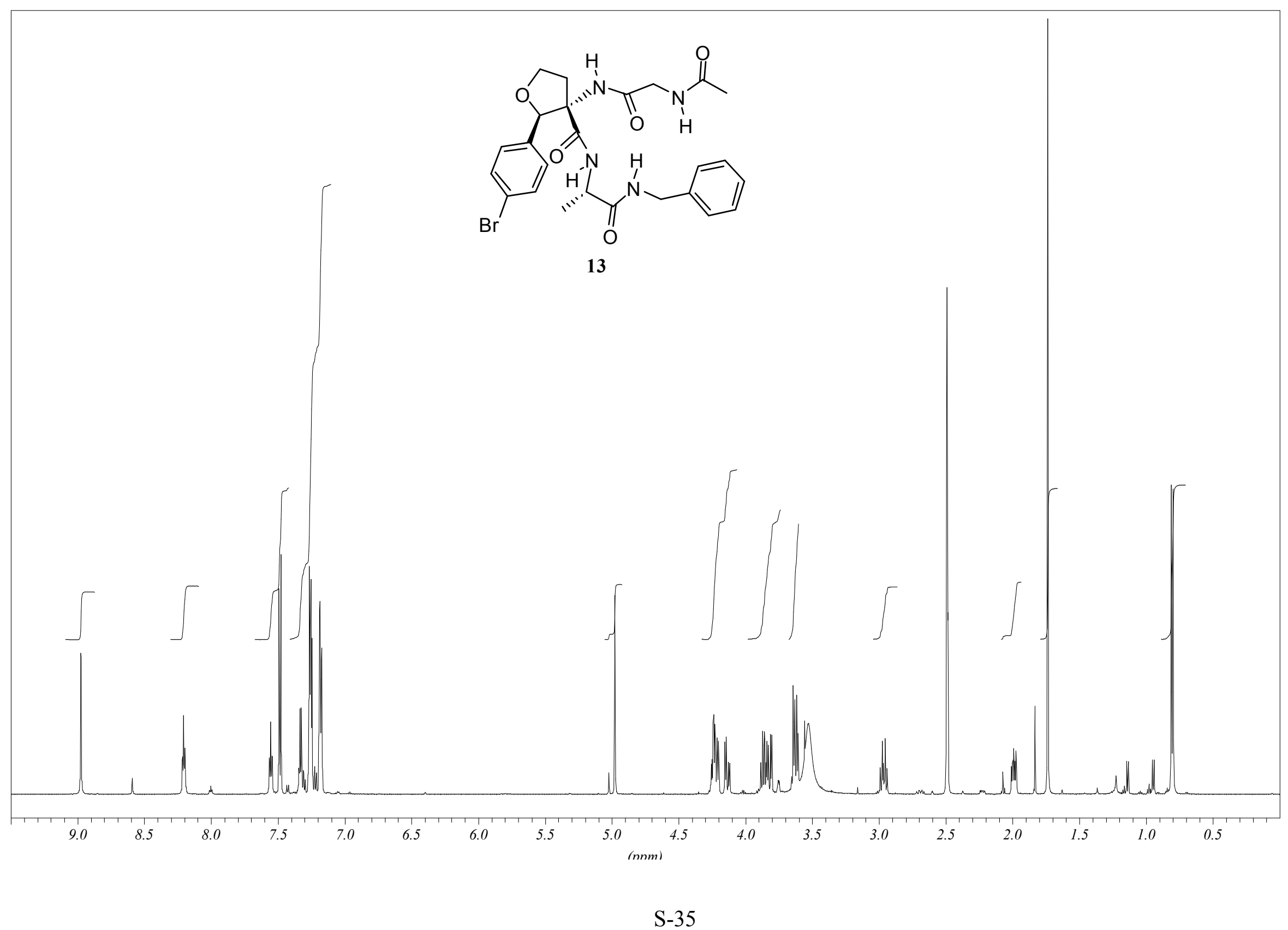




\section{4) Temperature dependence of NMR chemical shifts}

Temperature dependence of chemical shifts was measured to identify possible strong intramolecular hydrogen bonds in solution. The ${ }^{1} \mathrm{H}-\mathrm{NMR}$ spectra were recorded at various temperatures on a $600 \mathrm{MHz}$ spectrometer. Table 1 shows the determined chemical shift values (ppm) for each NH group of the examined compounds in the range of 293-373 K.

Table 1. Determined ${ }^{1} \mathrm{H}$ resonance chemical shift in $\mathrm{ppm}$ for $\mathrm{NH}$ protons at various temperature in [D6]-DMSO

A) Compound 13

\begin{tabular}{|c|c|c|c|c|}
\hline T[K]/ NH[ppm] & NHa & NHb & NHc & NHd \\
\hline $293 \mathrm{~K}$ & 8.245 & 9.027 & 7.356 & 7.558 \\
\hline $313 \mathrm{~K}$ & 8.137 & 8.886 & 7.288 & 7.550 \\
\hline $333 \mathrm{~K}$ & 8.026 & 8.738 & 7.225 & 7.537 \\
\hline $353 \mathrm{~K}$ & 7.924 & 8.593 & 7.160 & 7.520 \\
\hline $373 \mathrm{~K}$ & 7.819 & 8.446 & 7.097 & 7.497 \\
\hline
\end{tabular}

B) Compound 11

\begin{tabular}{|c|c|c|}
\hline T[K]/ NH[ppm] & NHa & NHb \\
\hline $293 \mathrm{~K}$ & 7.975 & 7.873 \\
\hline $313 \mathrm{~K}$ & 7.972 & 7.858 \\
\hline $333 \mathrm{~K}$ & 7.970 & 7.841 \\
\hline $353 \mathrm{~K}$ & 7.964 & 7.823 \\
\hline
\end{tabular}

The resonance values from table 1 were used to calculate the temperature dependence of the chemical shift. The measured values were plotted and fitted to a linear correlation function. From the plotted graph we calculated the corresponding temperature coefficient in $\mathrm{ppb} / \mathrm{K}$. These values are used to estimate the possibility of hydrogen bonds, using the following boundaries: Hydrogen bond very likely for values smaller than $-2 \mathrm{ppb} / \mathrm{K}$; intermediate range from -2 to $-3 \mathrm{ppb} / \mathrm{K}$ and no hydrogen bonding for values larger than $-4 \mathrm{ppb} / \mathrm{K}$. 
Figure S-5. Temperature dependence of amide proton resonances in dipeptide amide 11:
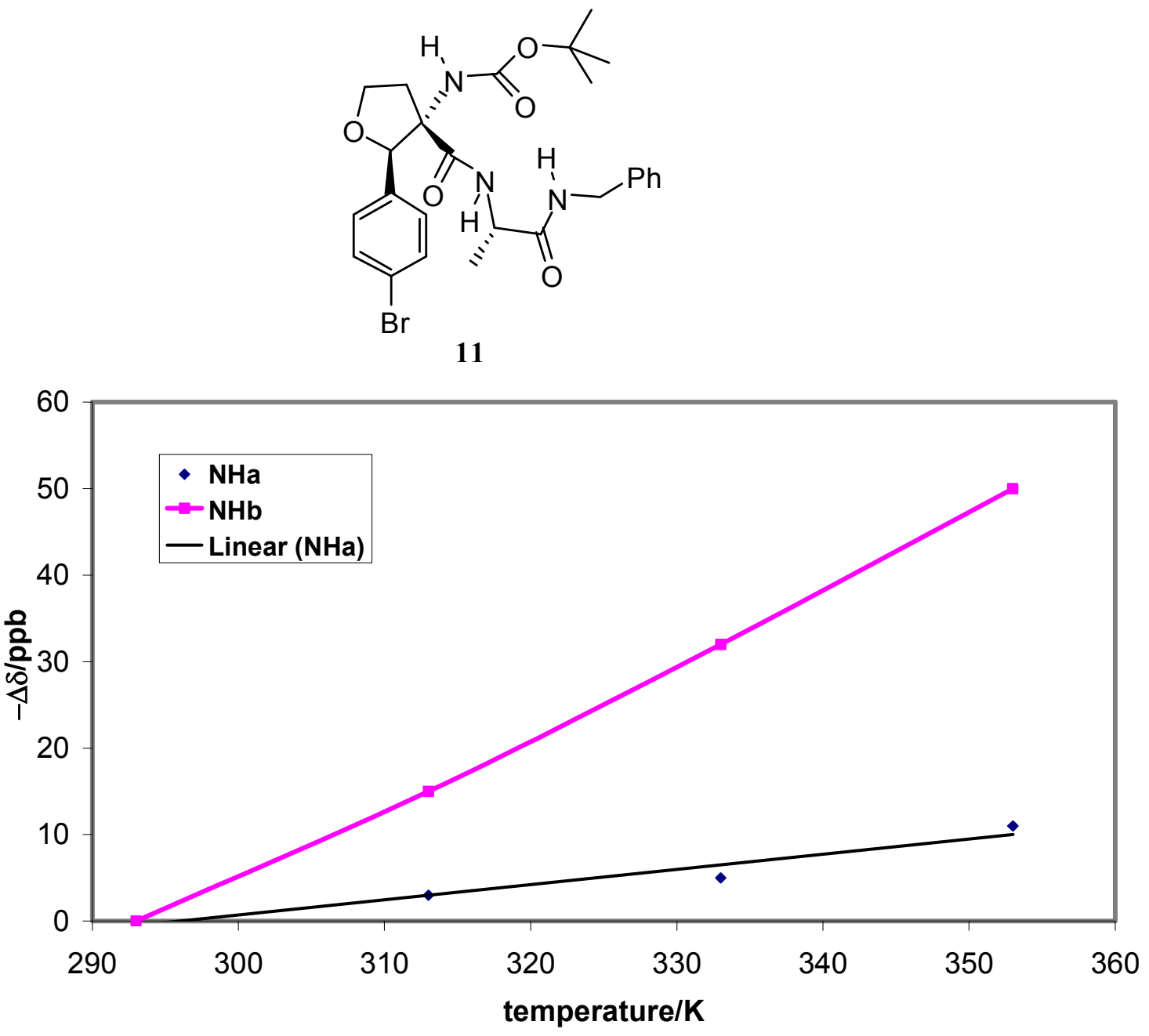

Figure S-6. Temperature dependence of amide proton resonances in tripeptide amide $\mathbf{1 3}$

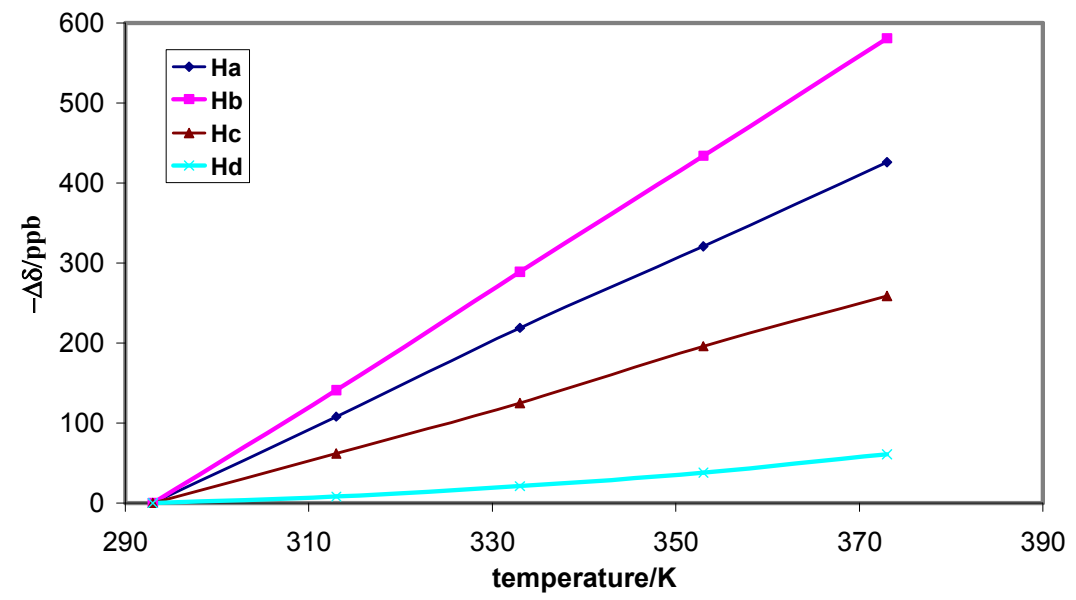

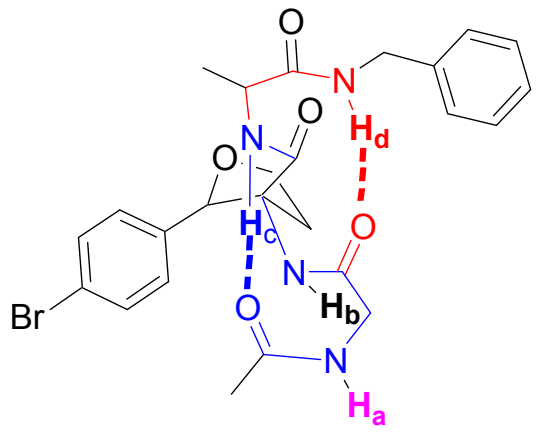




\section{NMR ROESY experiments}

1D-ROESY measurements in [D6]-DMSO was performed at $300 \mathrm{~K}$ on a Bruker DRX-600 spectrometer with a working frequency of $600.13 \mathrm{MHz}$. The difference ROESY experiment with selective excitation using the modified DPFGSE pulse sequence ( $\mathrm{q} 3$ Gaussian cascade Double Pulse Field Gradient Spin Echo) was used. For every irradiated proton a series of 5 experiments with different mixing times (from $10 \mathrm{~ms}$ to $1 \mathrm{~s}$ ) and with a relaxation delay of 2 s were acquired. An exponential window function with $4 \mathrm{~Hz}$ line broadening was applied before the Fourier transformation (FT) and a baseline correction was conducted after the FT. 2D-ROESY experiments with a mixing time of $500 \mathrm{~ms}$ were performed for structural calculations. Signal overlap restricts the number of observable intrastrand contacts. At least four ROESY interactions were detected for $\mathrm{NH}$ (13) and two for $\mathrm{NH}$ (16), as illustrated in Figure S-7.

Figure S-7. ROESY spectrum for compound 13 and illustration of observed contacts in solution.

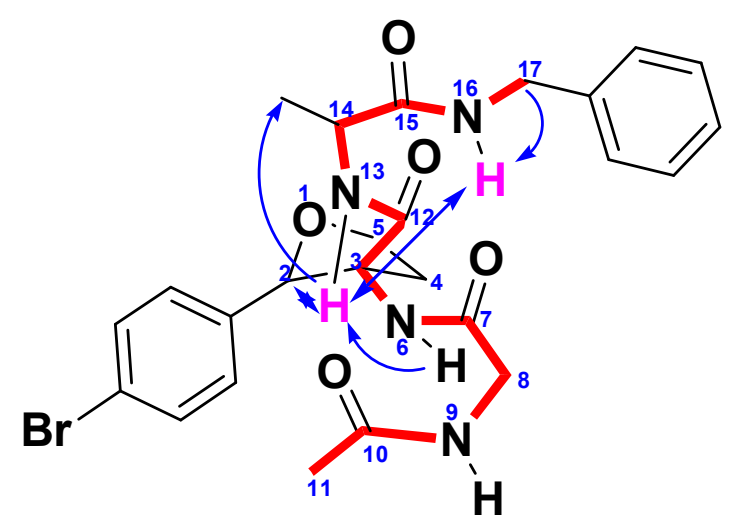

Compound 13 


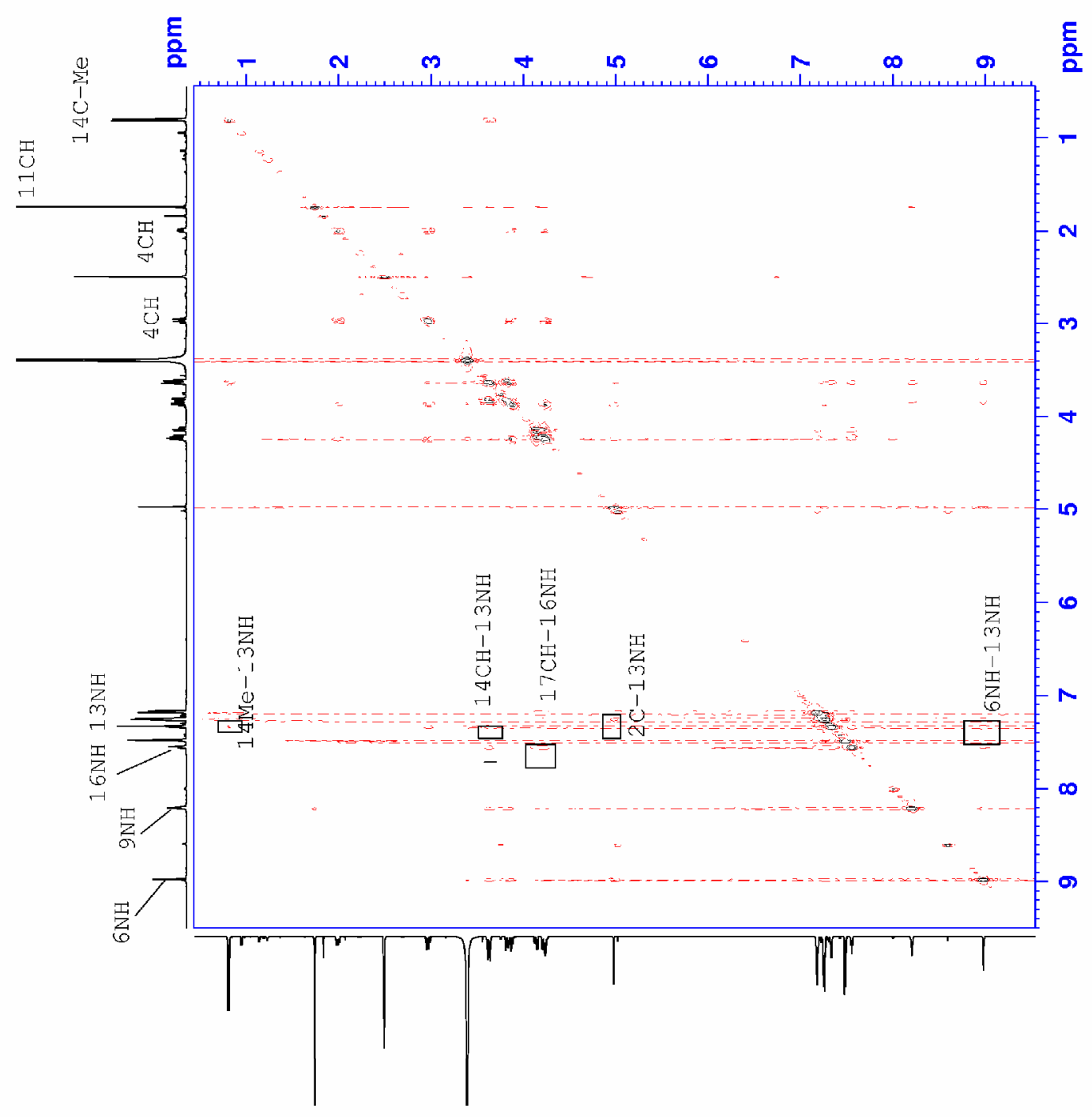

